\title{
The Geometry of Toric Hyperkähler Varieties
}

\author{
Hiroshi Konnd 1
}

\begin{abstract}
In this survey article we describe the geometry of toric hyperkähler varieties, which are hyperkähler quotients of the quaternionic vector spaces by tori. In particular, we discuss the Betti numbers, the cohomology ring, and variation of hyperkähler structures of these spaces with many improved results and proofs.
\end{abstract}

\section{Introduction}

Let a compact Lie group $G$ act on a hyperkähler manifold $M$, preserving its hyperkähler structure. Then we expect to define a quotient space of $M$ by the 'quaternionification' of $G$ so that the quotient space is also hyperkähler. Although we do not have a notion of 'quaternionification' of Lie groups, a hyperkähler quotient was introduced as such a kind of things. In HKLR a hyperkähler quotient was defined as an analogue of a quotient space in symplectic geometry. The geometry of hyperkähler quotients is a mixture of geometry of hyperkähler manifolds and symplectic quotients.

A hyperkähler structure is one of basic geometric structures in Riemannian geometry. A hyperkähler manifold is defined to be a Riemannian manifold whose holonomy group is contained in $S p\left(\frac{n}{4}\right)$, which is a group on Berger's list of Riemannian holonomy groups $[\mathrm{Ber}$. In other words, it is a Riemannian manifold with three complex structures, which satisfies the quaternion relation, and the Riemannian metric is Kähler with respect to each complex structure. Thus a hyperkähler structure is rather special. So hyperkähler manifolds have quite rich properties.

On the other hand, symplectic quotients also have rich properties. There are many techniques to investigate the topology of compact symplectic quotients. In particular, Kirwan proved the surjectivity of the Kirwan map, which is a map from the equivariant cohomology of the original space to the ordinary cohomology of its symplectic quotient. This enables one to compute the Betti numbers of symplectic quotients [Ki]. Moreover, in many cases a symplectic quotient can be identified with a quotient in geometric invariant theory (GIT for short). Due to this identification, one can study these quotients from viewpoints of both symplectic and algebraic geometry. Most fundamental examples are semi-projective toric varieties, which are symplectic quotients of the complex vector spaces by tori.

\footnotetext{
${ }^{1}$ Supported in part by the Grant-in-Aid for Scientific Research (C), No. 15540062.

2000 Mathematics Subject Classification: Primary 53C26; Secondary 53D20, 14L24
} 
In this article we describe the geometry of toric hyperkähler varieties, introduced by Bielawski and Dancer $[\mathrm{BD}$. A toric hyperkähler variety is defined to be a hyperkähler quotient of the quaternionic vector space by a torus. It is a non-compact hyperkähler variety on which a torus acts preserving its hyperkähler structure. It is a hyperkähler analogue of, but different from an ordinary toric variety. We explain fundamental results of toric hyperkähler varieties. We give many improved statements and proofs, which cannot be found in literatures. We refer the reader to $[\mathrm{P}$ for further results for toric hyperkähler varieties.

In Sections 2 we define toric hyperkähler varieties and introduce basic notations.

In Section 3 we prove basic properties of toric hyperkähler varieties. First we identify hyperkähler quotients with GIT quotients in our setting. Then we give characterizations of semi-stable points and closed orbits, which are very useful in this article. A toric hyperkähler variety has a parameter, which is shown to correspond to the period of its hyperkähler structure. Then we introduce natural morphisms between toric hyperkähler varieties with different parameters, influenced by [Kr, N1]. Then we show that a quotient for a generic parameter is an orbifold, and that its topology is independent of the parameter. A quotient for a certain parameter may have singularities which are worse than orbifold singularities.

In Section 4 we study the topology of toric hyperkähler varieties for generic parameters. Although a projective toric variety is determined by a convex polytope, a toric hyperkähler variety is determined by an arrangement of hyperplanes. We describe the Betti numbers and the cohomology ring in terms of the arrangement of hyperplanes, based on the works [BD] and [Ko1] respectively. We also describe another computation due to $[\mathrm{HS}$, where a toric hyperkähler variety is shown to be homotopy equivalent to a certain non-compact ordinary toric variety. We give a new proof of this homotopy equivalence. We also give another useful presentation of the cohomology ring, which is a simplification of the one in [Ko1]. In the case of hyperkähler quotients we have an analogue of the Kirwan map, but it is not yet known whether it is surjective or not in general. The above computation shows that the analogue of the Kirwan map is surjective in the case of toric hyperkähler varieties.

In Section 5 we give two examples, which illustrate the results in other sections.

In Section 6 we describe how a toric hyperkähler variety changes according to its period. In particular, we show that the so called wall-crossing phenomena are described as Mukai flops, which are special bimeromorphic transformations for hyperkähler varieties. We give a proof of this result using the technical tools developed in Section 3. This simplifies the proofs in Ko3.

The author would like to thank the organizers for the exciting workshop 'Toric Topology Conference 2006' at Osaka City University.

\section{Toric hyperkähler varieties}

Let $\mathbb{H}^{N}$ be the quaternionic vector space with the standard hyperkähler structure $\left(g, I_{1}, I_{2}, I_{3}\right)$. Here $g$ is the standard Riemannian metric on $\mathbb{H}^{N} \cong \mathbb{R}^{4 N}$, and the three complex structures $I_{1}, I_{2}, I_{3}$ are defined by the left multiplication by $I_{1}, I_{2}, I_{3}$, which are the standard generators of the quaternion $\mathbb{H}$. From now on we identify $\xi \in \mathbb{H}^{N}$ with $(z, w) \in \mathbb{C}^{N} \times \mathbb{C}^{N}$ by $\xi=z+w I_{2}$. The real torus $T^{N}=\{\zeta=$ $\left.\left(\zeta_{1}, \ldots, \zeta_{N}\right) \in \mathbb{C}^{N}|| \zeta_{i} \mid=1\right\}$ acts on $\mathbb{H}^{N}$ by $(z, w) \zeta=\left(z \zeta, w \zeta^{-1}\right)$, preserving its hyperkähler structure. Let $K$ be a connected subtorus of $T^{N}$ with the Lie algebra 
$k \subset t^{N}$. Then we have the following exact sequences;

$$
\begin{array}{llclclcll}
0 & \longrightarrow & k & \stackrel{\iota}{\longleftrightarrow} & t^{N} & \stackrel{\pi}{\longrightarrow} & t^{n} & \longrightarrow & \longrightarrow \\
0 & \longleftarrow & k^{*} & \stackrel{\iota^{*}}{\longleftarrow} & \left(t^{N}\right)^{*} \stackrel{\pi^{*}}{\longleftarrow} & \left(t^{n}\right)^{*} & \longleftarrow & &
\end{array}
$$

where $t^{n}=t^{N} / k$ is the Lie algebra of the quotient torus $T^{n}=T^{N} / K$. The action of $K$ on $\mathbb{H}^{N}$ admits a hyperkähler moment map

$$
\mu=\left(\mu_{1}, \mu_{2}, \mu_{3}\right): \mathbb{H}^{N} \rightarrow k^{*} \otimes \mathbb{R}^{3},
$$

which is given by

$$
\begin{aligned}
& \mu_{1}(z, w)=\pi \sum_{i=1}^{N}\left(\left|z_{i}\right|^{2}-\left|w_{i}\right|^{2}\right) \iota^{*} u_{i}, \\
& \left(\mu_{2}+\sqrt{-1} \mu_{3}\right)(z, w)=-2 \pi \sqrt{-1} \sum_{i=1}^{N} z_{i} w_{i} \iota^{*} u_{i},
\end{aligned}
$$

where $\left\{u_{1}, \ldots, u_{N}\right\} \subset\left(t^{N}\right)^{*}$ is the dual basis of the standard basis $\left\{X_{1}, \ldots, X_{N}\right\} \subset$ $t^{N}$. Note that the complex moment map $\mu_{\mathbb{C}}=\mu_{2}+\sqrt{-1} \mu_{3}: \mathbb{H}^{N} \rightarrow k_{\mathbb{C}}^{*}$ is holomorphic with respect to $I_{1}$, where $k_{\mathbb{C}}$ is the complexification of the Lie algebra $k$. Then Bielawski and Dancer introduced the following.

Definition 2.1 BD A toric hyperkähler variety $X(\alpha, \beta)$ is a hyperkähler quotient $\mu^{-1}(\alpha, \beta) / K$ for $(\alpha, \beta) \in k^{*} \times k_{\mathbb{C}}^{*}$.

Remark 2.2 Toric hyperkähler varieties are sometimes called 'hypertoric varieties' $[\mathrm{P}$. They are just the same objects.

A smooth part of $X(\alpha, \beta)$ is a $4 n$-dimensional hyperkähler manifold. We denote its hyperkähler structure by $\left(g, I_{1}, I_{2}, I_{3}\right)$ again. The torus $T^{n}=T^{N} / K$ acts on $X(\alpha, \beta)$, preserving its hyperkähler structure. Note that toric hyperkähler varieties are not toric varieties in the ordinary sense, but the corresponding objects in hyperkähler geometry to them.

Bielawski gave an intrinsic characterization of toric hyperkähler manifolds as follows.

Theorem 2.3 Bi] Let $M$ be a complete connected hyperkähler manifold of dimension $4 n$. Suppose that the torus $T^{n}$ acts on $M$ effectively, preserving the hyperkähler structure, and that $M$ has Euclidean volume growth. Then $M$ is isomorphic to one of toric hyperkähler manifolds in the sense of Definition 2.1 as a hyperkähler $T^{n}$ manifold.

\section{$3 \quad$ Holomorphic descriptions}

A toric hyperkähler variety $X(\alpha, \beta)=\mu_{1}^{-1}(\alpha) \cap \mu_{\mathbb{C}}^{-1}(\beta) / K$ can be considered as a symplectic quotient of $\mu_{\mathbb{C}}^{-1}(\beta)$ by the torus $K$. In many cases symplectic quotients can be identified with GIT quotients. In this section we describe this identification and apply it to prove basic properties of toric hyperkähler varieties. 


\subsection{Symplectic and GIT quotients}

Let $t_{\mathbb{Z}}^{N}=\sum_{i=1}^{N} \mathbb{Z} X_{i}$ and $\left(t_{\mathbb{Z}}^{N}\right)^{*}=\sum_{i=1}^{N} \mathbb{Z} u_{i}$ be the lattices in $t^{N}$ and $\left(t^{N}\right)^{*}$ respectively. We also set $k_{\mathbb{Z}}=k \cap t_{\mathbb{Z}}^{N}$ and $k_{\mathbb{Z}}^{*}=H o m_{\mathbb{Z}}\left(k_{\mathbb{Z}}, \mathbb{Z}\right)$. The element $\alpha \in k_{\mathbb{Z}}^{*}$ induces the character $\chi_{\alpha}: K_{\mathbb{C}} \rightarrow \mathbb{C}^{\times}$, where $K_{\mathbb{C}}$ is the complexfication of $K$. Let $L^{\otimes m}=\mathbb{H}^{N} \times \mathbb{C}$ be the trivial holomorphic line bundle on which $K_{\mathbb{C}}$ acts by

$$
((z, w), v)_{m} \zeta=\left(\left(z \zeta, w \zeta^{-1}\right), v \chi_{\alpha}(\zeta)^{m}\right)_{m} .
$$

Let us consider the GIT quotient of the affine variety $\mu_{\mathbb{C}}^{-1}(\beta)$ by $K_{\mathbb{C}}$ with respect to the linearization induced by $\alpha \in k_{\mathbb{Z}}^{*}$. Recall that a point $(z, w) \in \mu_{\mathbb{C}}^{-1}(\beta)$ is $\alpha$ semi-stable if and only if there exists $m \in \mathbb{Z}_{>0}$ and a polynomial $f(p, q)$, where $p, q \in \mathbb{C}^{N}$, such that $f((p, q) \zeta)=f(p, q) \chi_{\alpha}(\zeta)^{m}$ for any $\zeta \in K_{\mathbb{C}}$ and $f(z, w) \neq 0$. We denote the set of $\alpha$-semi-stable points in $\mu_{\mathbb{C}}^{-1}(\beta)$ by $\mu_{\mathbb{C}}^{-1}(\beta)^{\alpha-s s}$. Then we have a categorical quotient $\phi: \mu_{\mathbb{C}}^{-1}(\beta)^{\alpha-s s} \rightarrow \mu_{\mathbb{C}}^{-1}(\beta)^{\alpha-s s} / / K_{\mathbb{C}}$. Here we summarize the properties of categorical quotients. We refer the reader to Do, MFK] for the proof.

Lemma 3.1 For any point $p \in \mu_{\mathbb{C}}^{-1}(\beta)^{\alpha-s s} / / K_{\mathbb{C}}$, the fiber $\phi^{-1}(p)$ consists of

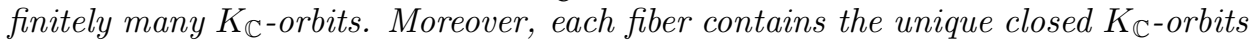
in $\mu_{\mathbb{C}}^{-1}(\beta)^{\alpha-s s}$. Thus the categorical quotient $\mu_{\mathbb{C}}^{-1}(\beta)^{\alpha-s s} / / K_{\mathbb{C}}$ can be identified with a set of closed $K_{\mathbb{C}}$-orbits in $\mu_{\mathbb{C}}^{-1}(\beta)^{\alpha-s s}$.

Define a Hermitian metric on the holomorphic line bundle $L^{\otimes m}=\mathbb{H}^{N} \times \mathbb{C}$ by $\left\|((z, w), v)_{m}\right\|=|v| e^{-\frac{\pi}{2} m\left(|z|^{2}+|w|^{2}\right)}$. It induces the Chern connection $\nabla^{\otimes m}$ on $L^{\otimes m}$. Note that the first Chern form $c_{1}\left(\nabla^{\otimes m}\right)=m \omega_{1}$, where $\omega_{1} \in \Omega^{2}\left(\mathbb{H}^{N}\right)$ is the standard Kähler form with respect to $I_{1}$. Since the action of the real torus $K$ on $L^{\otimes m}$ preserves the holomorphic structure and the Hermitian metric, it also preserves the Chern connection $\nabla^{\otimes m}$. Let us introduce the following function. Fix $(z, w) \in \mathbb{H}^{N}$. Then define $l_{(z, w)}: K_{\mathbb{C}} \rightarrow \mathbb{R}$ by

$$
l_{(z, w)}(\zeta)=\frac{1}{2 \pi} \log \frac{\left\|((z, w), v)_{-1} \zeta\right\|}{\left\|((z, w), v)_{-1}\right\|} .
$$

In particular, for $X \in k$ and $t \in \mathbb{R}$, we have

$$
\begin{aligned}
& l_{(z, w)}(\operatorname{Exp} \sqrt{-1} t X)=\langle\alpha, t X\rangle \\
& \quad+\frac{1}{4} \sum_{i=1}^{N}\left|z_{i}\right|^{2} e^{-4 \pi\left\langle u_{i}, t X\right\rangle}+\frac{1}{4} \sum_{i=1}^{N}\left|w_{i}\right|^{2} e^{4 \pi\left\langle u_{i}, t X\right\rangle}-\frac{1}{4}\left(|z|^{2}+|w|^{2}\right),
\end{aligned}
$$

where Exp: $k_{\mathbb{C}} \rightarrow K_{\mathbb{C}}$ is the exponential map. Then we have the following.

Lemma $3.2(1) l_{(z, w)}$ is a convex function.

(2) $\zeta$ is a critical point if and only if $\mu_{1}((z, w) \zeta)=\alpha$.

(3) All critical points are minima of $l_{(z, w)}$.

(4) The following ( $i),($ ii) and (iii) are equivalent:

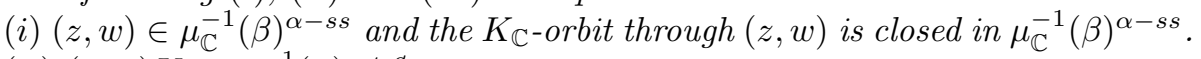

(ii) $(z, w) K_{\mathbb{C}} \cap \mu_{1}^{-1}(\alpha) \neq \emptyset$.

(iii) $l_{(z, w)}$ attains a minimum.

Proof. A direct computation shows

$$
\begin{aligned}
& \left.\frac{d}{d t} l_{(z, w)}(\operatorname{Exp} \sqrt{-1} t X)\right|_{t=t_{0}}=\left\langle\alpha-\mu_{1}\left((z, w) \operatorname{Exp} \sqrt{-1} t_{0} X\right), X\right\rangle, \\
& \left.\frac{d^{2}}{d t^{2}} l_{(z, w)}(\operatorname{Exp} \sqrt{-1} t X)\right|_{t=t_{0}}=\left|X^{*}\right|_{\text {at }(z, w) \operatorname{Exp} \sqrt{-1} t_{0} X}^{2},
\end{aligned}
$$


where $X^{*}$ is a vector field on $\mu_{\mathbb{C}}^{-1}(\beta)$ induced by $X \in k$. Then it is easy to see (1), (2) and (3). We refer the reader to [N2] for the proof of (4).

By using Lemmas 3.1 and 3.2 , we can show the following.

Proposition 3.3 If we fix $\alpha \in k_{\mathbb{Z}}^{*}$, then the natural map $\iota: X(\alpha, \beta) \rightarrow \mu_{\mathbb{C}}^{-1}(\beta)^{\alpha-s s} / / K_{\mathbb{C}}$ is a homeomorphism.

Thus we can identify the symplectic quotient $\left(X(\alpha, \beta), I_{1}\right)$ with the GIT quotient $\mu_{\mathbb{C}}^{-1}(\beta)^{\alpha-s s} / / K_{\mathbb{C}}$. This principle was established in $\mathrm{KN}$, MFK in the case of projective quotients. The above version is proved in [N2].

\subsection{Characterization of semi-stable points and closed orbits}

The following technical lemma will be very useful in this article.

Lemma 3.4 Suppose that $\alpha \in k_{\mathbb{Z}}^{*}$.

(1) A point $(z, w) \in \mu_{\mathbb{C}}^{-1}(\beta)$ is $\alpha$-semi-stable if and only if

$$
\alpha \in \sum_{i=1}^{N} \mathbb{R}_{\geq 0}\left|z_{i}\right|^{2} \iota^{*} u_{i}+\sum_{i=1}^{N} \mathbb{R}_{\geq 0}\left|w_{i}\right|^{2}\left(-\iota^{*} u_{i}\right) .
$$

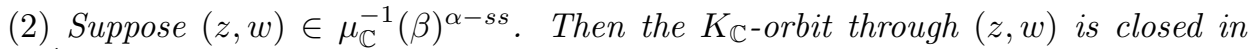
$\mu_{\mathbb{C}}^{-1}(\beta)^{\alpha-s s}$ if and only if

$$
\alpha \in \sum_{i=1}^{N} \mathbb{R}_{>0}\left|z_{i}\right|^{2} \iota^{*} u_{i}+\sum_{i=1}^{N} \mathbb{R}_{>0}\left|w_{i}\right|^{2}\left(-\iota^{*} u_{i}\right) .
$$

Proof. (1) Suppose $(z, w) \in \mu_{\mathbb{C}}^{-1}(\beta)^{\alpha-s s}$. Then there exists $m \in \mathbb{Z}_{>0}$ and a polynomial $f\left(p_{1}, \ldots, p_{N}, q_{1}, \ldots q_{N}\right)$ such that $f((p, q) \zeta)=f(p, q) \chi_{\alpha}(\zeta)^{m}$ for any $\zeta \in K_{\mathbb{C}}$ and $f(z, w) \neq 0$. So there exists a monomial $f_{0}(p, q)=\prod_{i=1}^{N} p_{i}^{a_{i}} \prod_{i=1}^{N} q_{i}^{b_{i}}$, where $a_{i}, b_{i} \in \mathbb{Z}_{\geq 0}$, such that $f_{0}((p, q) \zeta)=f_{0}(p, q) \chi_{\alpha}(\zeta)^{m}$ and $f_{0}(z, w) \neq 0$. The second condition implies that $a_{i}=0$ if $z_{i}=0$, and $b_{i}=0$ if $w_{i}=0$. Moreover, the first condition implies $m \alpha=\sum_{i=1}^{N} a_{i} \iota^{*} u_{i}+\sum_{i=1}^{N} b_{i}\left(-\iota^{*} u_{i}\right)$. Thus we proved (3.3).

Suppose that (3.3) holds. Then there exists $m \in \mathbb{Z}_{>0}$ and $a_{i}, b_{i} \in \mathbb{Z}_{\geq 0}$ such that $m \alpha=\sum_{i=1}^{N} a_{i} \iota^{*} u_{i}+\sum_{i=1}^{N} b_{i}\left(-\iota^{*} u_{i}\right)$ and such that $a_{i}=0$ if $z_{i}=0$, and $b_{i}=0$ if $w_{i}=0$. Then the monomial $f_{0}(p, q)=\prod_{i=1}^{N} p_{i}^{a_{i}} \prod_{i=1}^{N} q_{i}^{b_{i}}$ satisfies $f_{0}((p, q) \zeta)=$ $f_{0}(p, q) \chi_{\alpha}(\zeta)^{m}$ and $f_{0}(z, w) \neq 0$. Therefore we have $(z, w) \in \mu_{\mathbb{C}}^{-1}(\beta)^{\alpha-s s}$.

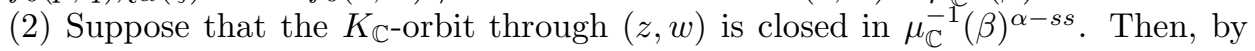
Lemma 3.2 there exists $\zeta \in K_{\mathbb{C}}$ such that $\alpha=\mu_{1}((z, w) \zeta)$. Moreover, we have $\mu_{1}((z, w) \zeta) \in \sum_{i=1}^{N} \mathbb{R}_{>0}\left|z_{i}\right|^{2} \iota^{*} u_{i}+\sum_{i=1}^{N} \mathbb{R}_{>0}\left|w_{i}\right|^{2}\left(-\iota^{*} u_{i}\right)$. This implies (3.4).

Suppose that (3.4) holds. By Lemma 3.2, we have only to show that $l_{(z, w)}$ attains a minimum. We claim that

$$
\lim _{t \rightarrow \infty} l_{(z, w)}(\operatorname{Exp} \sqrt{-1} t X) \neq \infty \text { if and only if } X \in k_{(z, w)}
$$

where $k_{(z, w)}$ is the Lie algebra of the isotropy subgroup $K_{(z, w)}$ of $K$ at $(z, w)$. In fact, by (3.1), the condition $\lim _{t \rightarrow \infty} l_{(z, w)}(\operatorname{Exp} \sqrt{-1} t X) \neq \infty$ implies the following conditions $(i)$ and $(i i)$;

(i) $\langle\alpha, X\rangle \leq 0$

(ii) $\left\langle u_{i}, X\right\rangle \geq 0$ if $z_{i} \neq 0$, and $\left\langle u_{i}, X\right\rangle \leq 0$ if $w_{i} \neq 0$. 
Due to the assumption (3.4), (ii) implies $\langle\alpha, X\rangle \geq 0$. Due to $(i)$, we conclude that $\left\langle u_{i}, X\right\rangle=0$ if $z_{i} \neq 0$, and that $\left\langle u_{i}, X\right\rangle=0$ if $w_{i} \neq 0$. This implies that $X \in k_{(z, w)}$. On the other hand, it is easy to see that, if $X \in k_{(z, w)}$, then $l_{(z, w)}(\operatorname{Exp} \sqrt{-1} t X)$ is independent of $t \in \mathbb{R}$. Thus we proved the claim (3.5). Then, using the convexity of $l_{(z, w)}$, we can easily show that $l_{(z, w)}$ attains a minimum.

By this lemma the notion of $\alpha$-semi-stability is defined for any $\alpha \in k^{*}$. Namely, a point $(z, w) \in \mu_{\mathbb{C}}^{-1}(\beta)$ is defined to be $\alpha$-semi-stable if and only if

$$
\alpha \in \sum_{i=1}^{N} \mathbb{R}_{\geq 0}\left|z_{i}\right|^{2} \iota^{*} u_{i}+\sum_{i=1}^{N} \mathbb{R}_{\geq 0}\left|w_{i}\right|^{2}\left(-\iota^{*} u_{i}\right)
$$

for any $\alpha \in k^{*}$. Moreover, since the above argument works for any $\alpha \in k^{*}$, we can identify a toric hyperkähler variety $\left(X(\alpha, \beta), I_{1}\right)$ with $\mu_{\mathbb{C}}^{-1}(\beta)^{\alpha-s s} / / K_{\mathbb{C}}$ for any $\alpha \in k^{*}$.

Applying this lemma to the case $\alpha=0 \in k^{*}$, we have $\mu_{\mathbb{C}}^{-1}(\beta)^{0-s s}=\mu_{\mathbb{C}}^{-1}(\beta)$. So the toric hyperkähler variety $X(0, \beta)=\mu_{\mathbb{C}}^{-1}(\beta) / / K_{\mathbb{C}}$ is an affine variety.

\subsection{Natural Morphisms}

The inclusion $\mu_{\mathbb{C}}^{-1}(\beta)^{\alpha-s s} \subset \mu_{\mathbb{C}}^{-1}(\beta)$ induces a natural morphism from a GIT quotient $\mu_{\mathbb{C}}^{-1}(\beta)^{\alpha-s s} / / K_{\mathbb{C}}$ to an affine quotient $\mu_{\mathbb{C}}^{-1}(\beta) / / K_{\mathbb{C}}$, which we denote by

$$
\pi_{1}:\left(X(\alpha, \beta), I_{1}\right) \rightarrow\left(X(0, \beta), I_{1}\right) .
$$

If we denote $X(\alpha, \beta)$ by $X\left(\alpha_{1}, \alpha_{2}, \alpha_{3}\right)$, where $(\alpha, \beta)=\left(\alpha_{1}, \alpha_{2}+\sqrt{-1} \alpha_{3}\right)$, then the natural morphism is written as $\pi_{1}:\left(X\left(\alpha_{1}, \alpha_{2}, \alpha_{3}\right), I_{1}\right) \rightarrow\left(X\left(0, \alpha_{2}, \alpha_{3}\right), I_{1}\right)$. By rotating the complex structures $I_{1}, I_{2}$ and $I_{3}$ cyclically, we also have the natural morphisms

$$
\begin{aligned}
& \pi_{2}:\left(X\left(\alpha_{1}, \alpha_{2}, \alpha_{3}\right), I_{2}\right) \rightarrow\left(X\left(\alpha_{1}, 0, \alpha_{3}\right), I_{2}\right), \\
& \pi_{3}:\left(X\left(\alpha_{1}, \alpha_{2}, \alpha_{3}\right), I_{3}\right) \rightarrow\left(X\left(\alpha_{1}, \alpha_{2}, 0\right), I_{3}\right) .
\end{aligned}
$$

The following is a basic property of the natural morphisms, which will be useful in the next section.

Proposition 3.5 The natural morphism $\pi_{1}:\left(X(\alpha, \beta), I_{1}\right) \rightarrow\left(X(0, \beta), I_{1}\right)$ is proper and surjective for any $(\alpha, \beta) \in k^{*} \times k_{\mathbb{C}}^{*}$.

Proof. First we prove that $\pi_{1}$ is proper. Suppose the contrary. Namely, there exists a compact set $S \subset X(0, \beta)$ such that $\pi_{1}^{-1}(S) \subset X(\alpha, \beta)$ is non-compact. Then we can take a sequence $\left\{p_{(i)}\right\} \subset \mu^{-1}(\alpha, \beta)$ such that $\left[p_{(i)}\right] \in \pi^{-1}(S)$ and $\left\|p_{(i)}\right\| \rightarrow \infty$. If we set $p_{(i)}^{\prime}=\frac{p_{(i)}}{\left\|p_{(i)}\right\|} \in \mu^{-1}\left(\frac{\alpha}{\left\|p_{(i)}\right\|^{2}}, \frac{\beta}{\left\|p_{(i)}\right\|^{2}}\right)$, then we may assume that $\left\{p_{(i)}^{\prime}\right\}$ converges to $p_{(\infty)}^{\prime} \in \mathbb{H}^{N}$. Then we have $\left\|p_{(\infty)}^{\prime}\right\|=1$ and $p_{(\infty)}^{\prime} \in \mu^{-1}(0,0)$.

On the other hand, we can take $q_{(i)} \in \mu^{-1}(0, \beta)$ such that $\pi_{1}\left(\left[p_{(i)}\right]\right)=\left[q_{(i)}\right]$. Since $\left\{\left[q_{(i)}\right]\right\} \subset S,\left\{q_{(i)}\right\}$ is bounded in $\mu^{-1}(0, \beta)$. If we set $q_{(i)}^{\prime}=\frac{q_{(i)}}{\left\|p_{(i)}\right\|} \in$ $\mu^{-1}\left(0, \frac{\beta}{\left\|p_{(i)}\right\|^{2}}\right)$, then $\left\{q_{(i)}^{\prime}\right\}$ converges to $(0,0) \in \mathbb{H}^{N}$. Let $\pi_{1}^{\prime}: X\left(\frac{\alpha}{\left\|p_{(i)}\right\|^{2}}, \frac{\beta}{\left\|p_{(i)}\right\|^{2}}\right) \rightarrow$ $X\left(0, \frac{\beta}{\left\|p_{(i)}\right\|^{2}}\right)$ be the natural morphism. Since $\pi_{1}^{\prime}\left(\left[p_{(i)}^{\prime}\right]\right)=\left[q_{(i)}^{\prime}\right]$, we have $q_{(i)}^{\prime} \in$ $\overline{p_{(i)}^{\prime} K_{\mathbb{C}}}$. Therefore we can take $\left\{\zeta_{(i)}\right\} \subset K_{\mathbb{C}}$ such that $\left\{p_{(i)}^{\prime} \zeta_{(i)}\right\}$ converges to $(0,0)$. Then we can show that $\left\{p_{(\infty)}^{\prime} \zeta_{(i)}\right\}$ converges to $(0,0)$. So we have $(0,0) \in \overline{p_{(\infty)}^{\prime} K_{\mathbb{C}}}$.

However, since $p_{(\infty)}^{\prime} \in \mu^{-1}(0,0)$, we have $\overline{p_{(\infty)}^{\prime} K_{\mathbb{C}}}=p_{(\infty)}^{\prime} K_{\mathbb{C}}$. Therefore we have $p_{(\infty)}^{\prime}=(0,0)$. This contradicts to $\left\|p_{(\infty)}^{\prime}\right\|=1$. 
Surjectivity of the map $\pi_{1}$ will be proved at the end of Subsection 4.1 .

The above proof is essentially the same as the one in $\mathrm{Kr}$, $\mathrm{N1}$, where the similar result is proved in the case of quiver varieties. See also $\mathrm{AO}$ for the proof.

Let $\left(k^{*} \times k_{\mathbb{C}}^{*}\right)_{\text {reg }}$ be the set of regular values of the hyperkähler moment map $\mu=\left(\mu_{1}, \mu_{\mathbb{C}}\right): \mathbb{H}^{N} \rightarrow k^{*} \otimes \mathbb{R}^{3} \cong k^{*} \times k_{\mathbb{C}}^{*}$. Denote the isotropy subgroup of $K$ at $(z, w) \in \mathbb{H}^{N}$ by $K_{(z, w)}$. Set

$$
\begin{aligned}
& \Lambda=\left\{K_{(z, w)} \mid(z, w) \in \mathbb{H}^{N}\right\}, \\
& \Lambda^{(d)}=\{H \in \Lambda \mid \operatorname{dim} H=d\} \text { for } d=0, \ldots, N-n .
\end{aligned}
$$

We write $\Lambda^{(1)}=\left\{H_{1}^{(1)}, \ldots, H_{l}^{(1)}\right\}$. The subspace of codimension one

$$
W_{s}=\left\{v \in k^{*} \mid\left\langle v, \operatorname{Lie} H_{s}^{(1)}\right\rangle=0\right\} \subset k^{*}
$$

is called a wall. Note that the wall $W_{s}$ is spanned by $\left\{\iota^{*} u_{i} \mid\left\langle\iota^{*} u_{i}, \operatorname{Lie} H_{s}^{(1)}\right\rangle=0\right\}$. Moreover, we have the following.

Proposition $3.6(1)\left(k^{*} \times k_{\mathbb{C}}^{*}\right)_{r e g}=k^{*} \times k_{\mathbb{C}}^{*} \backslash \bigcup_{s=1}^{l} W_{s} \otimes W_{s \mathbb{C}}$

(2) If $(\alpha, \beta) \in\left(k^{*} \times k_{\mathbb{C}}^{*}\right)_{\text {reg }}$, then $X(\alpha, \beta)$ is an orbifold.

(3) If $(0, \beta) \in\left(k^{*} \times k_{\mathbb{C}}^{*}\right)_{\text {reg }}$, then $\pi_{1}:\left(X(\alpha, \beta), I_{1}\right) \rightarrow\left(X(0, \beta), I_{1}\right)$ is a biholomorphic map for any $\alpha \in k^{*}$.

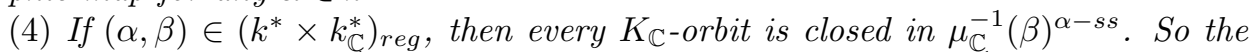
categorical quotient $\mu_{\mathbb{C}}^{-1}(\beta)^{\alpha-s s} / / K_{\mathbb{C}}$ is a geometric quotient $\mu_{\mathbb{C}}^{-1}(\beta)^{\alpha-s s} / K_{\mathbb{C}}$.

(5) The topology of $X(\alpha, \beta)$ is independent of $(\alpha, \beta)$ as far as $(\alpha, \beta) \in\left(k^{*} \times k_{\mathbb{C}}^{*}\right)_{\text {reg }}$. That is, all the $X(\alpha, \beta)$ are diffeomorphic to each other as far as $(\alpha, \beta) \in\left(k^{*} \times\right.$ $\left.k_{\mathbb{C}}^{*}\right)_{\text {reg. }}$. The diffeomorphisms are given by compositions of natural morphisms.

Proof. (1) Let $f: \mathbb{H} \rightarrow \mathbb{R} \times \mathbb{C}$ be a map defined by $f(a, b)=\left(\pi\left(|a|^{2}-|b|^{2}\right),-2 \pi \sqrt{-1} a b\right)$. We can easily observe that $(a, b) \in \mathbb{C} \times \mathbb{C} \cong \mathbb{H}$ is a regular point of $f$ if and only if $(a, b) \neq(0,0)$. Since $\left(d \mu_{K}\right)_{(z, w)}=\sum_{i=1}^{N}(d f)_{\left(z_{i}, w_{i}\right)} \otimes \iota^{*} u_{i} \in k^{*} \times k_{\mathbb{C}}^{*},(z, w) \in \mathbb{H}^{N}$ is a critical point of $\mu_{K}$ if and only if $\operatorname{span}\left\{\iota^{*} u_{i} \mid\left(z_{i}, w_{i}\right) \neq(0,0)\right\} \varsubsetneqq k^{*}$. This implies (1).

(2) is clear. We omit the proof.

(3) If $(0, \beta) \in\left(k^{*} \times k_{\mathbb{C}}^{*}\right)_{r e g}$, then $\operatorname{span}\left\{\iota^{*} u_{i} \mid z_{i} w_{i} \neq 0\right\}=k^{*}$ for any $(z, w) \in \mu_{\mathbb{C}}^{-1}(\beta)$.

By Lemma 3.4 we have $\mu_{\mathbb{C}}^{-1}(\beta)^{\alpha-s s}=\mu_{\mathbb{C}}^{-1}(\beta)$. So we have proved (3).

(4) Suppose the contrary. Namely, there exists $(z, w) \in \mu_{\mathbb{C}}^{-1}(\beta)^{\alpha-s s}$ such that $(z, w) K_{\mathbb{C}}$ is not closed in $\mu_{\mathbb{C}}^{-1}(\beta)^{\alpha-s s}$. Then there exists $\left(z^{\prime}, w^{\prime}\right) \in \overline{(z, w) K_{\mathbb{C}}} \cap$ $\mu_{\mathbb{C}}^{-1}(\beta)^{\alpha-s s}$ such that $\left(z^{\prime}, w^{\prime}\right) K_{\mathbb{C}}$ is closed in $\mu_{\mathbb{C}}^{-1}(\beta)^{\alpha-s s}$. By Lemma 3.2 we may assume $\left(z^{\prime}, w^{\prime}\right) \in \mu^{-1}(\alpha, \beta)$. Since $\operatorname{dim} K_{\left(z^{\prime}, w^{\prime}\right)} \geq 1$, we can easily see that $\left(z^{\prime}, w^{\prime}\right)$ is a critical point of $\mu$. Therefore $(\alpha, \beta)$ is a critical value of $\mu$. This is a contradiction.

(5) We denote $X(\alpha, \beta)$ by $X\left(\alpha_{1}, \alpha_{2}, \alpha_{3}\right)$, where $(\alpha, \beta)=\left(\alpha_{1}, \alpha_{2}+\sqrt{-1} \alpha_{3}\right)$. Take $\left(\alpha_{1}, \alpha_{2}, \alpha_{3}\right),\left(\alpha_{1}^{\prime}, \alpha_{2}^{\prime}, \alpha_{3}^{\prime}\right) \in\left(k^{*} \times k_{\mathbb{C}}^{*}\right)_{r e g}$. We have to show that $X\left(\alpha_{1}, \alpha_{2}, \alpha_{3}\right)$ is diffeomorphic to $X\left(\alpha_{1}^{\prime}, \alpha_{2}^{\prime}, \alpha_{3}^{\prime}\right)$. By Lemma 3.4, we may assume that $\left(0,0, \alpha_{3}\right)$ and $\left(0, \alpha_{2}^{\prime}, 0\right)$ are in $\left(k^{*} \times k_{\mathbb{C}}^{*}\right)_{\text {reg }}$. Then $X\left(\alpha_{1}, \alpha_{2}, \alpha_{3}\right)$ is diffeomorphic to $X\left(\alpha_{1}^{\prime}, \alpha_{2}, \alpha_{3}\right)$, because $\left(0, \alpha_{2}+\sqrt{-1} \alpha_{3}\right) \in\left(k^{*} \times k_{\mathbb{C}}^{*}\right)_{\text {reg }}$. By rotating $I_{1}, I_{2}$ and $I_{3}$ cyclically, we see that $X\left(\alpha_{1}^{\prime}, \alpha_{2}, \alpha_{3}\right)$ is diffeomorphic to $X\left(\alpha_{1}^{\prime}, \alpha_{2}^{\prime}, \alpha_{3}\right)$ and also that $X\left(\alpha_{1}^{\prime}, \alpha_{2}^{\prime}, \alpha_{3}\right)$ is diffeomorphic to $X\left(\alpha_{1}^{\prime}, \alpha_{2}^{\prime}, \alpha_{3}^{\prime}\right)$. Thus we finish the proof.

It is easy to see when a toric hyperkähler orbifold $X(\alpha, \beta)$ is a manifold. 
Proposition 3.7 Fix $(\alpha, \beta) \in\left(k^{*} \times k_{\mathbb{C}}^{*}\right)_{\text {reg }}$. Then the subtorus $K$ acts on $\mu^{-1}(\alpha, \beta)$ freely if and only if

$$
t_{\mathbb{Z}}^{N}=k_{\mathbb{Z}}+\sum_{i \notin J} \mathbb{Z} X_{j} \quad \text { as a } \mathbb{Z} \text {-module }
$$

holds for any $J \subset\{1, \ldots, N\}$ such that $\left\{\iota^{*} u_{i} \mid i \in J\right\}$ forms a basis of $k^{*}$.

See Ko1 for the proof. Note that (3.6) is the condition for the subtorus $K$. It is independent of $(\alpha, \beta)$.

\section{Topology}

In this section we discuss the topology of toric hyperkähler orbifolds, based on the works $\mathrm{BD}, \mathrm{Ko1}, \mathrm{HS}$. It describes similarities and differences between the topology of ordinary toric orbifolds and toric hyperkähler orbifolds very well.

\subsection{The Core}

Let $\Theta$ be the set of all maps from $\{1, \ldots, N\}$ to $\{1,-1\}$. For each $\epsilon \in \Theta$, we define

$$
V_{\epsilon}=\left\{(z, w) \in \mathbb{H}^{N} \mid w_{i}=0 \text { if } \epsilon(i)=1 \text {, and } z_{i}=0 \text { if } \epsilon(i)=-1 \text { for } i=1, \ldots, N\right\} .
$$

Note that $V_{\epsilon} \cong \mathbb{C}^{N}$ and $V_{\epsilon} \subset \mu_{\mathbb{C}}^{-1}(0)$. If we fix $\alpha \in k^{*}$, then

$$
M_{\epsilon}(\alpha)=\left\{V_{\epsilon} \cap \mu_{1}^{-1}(\alpha)\right\} / K \subset X(\alpha, 0)
$$

is an ordinary toric variety. Moreover, we set

$$
\Theta_{c p t}(\alpha)=\left\{\epsilon \in \Theta \mid M_{\epsilon}(\alpha) \text { is compact }\right\} .
$$

Then we have the following.

Proposition 4.1 Fix $\alpha \in k^{*}$. Let $\pi_{1}: X(\alpha, 0) \rightarrow X(0,0)$ be the natural morphism. Then the following holds.

(1) $\pi_{1}^{-1}([0,0])$ is a $T^{n}$-equivariant deformation retract of $X(\alpha, 0)$.

(2) In addition, suppose that $\iota^{*} u_{i} \neq 0$ for any $i=1, \ldots, N$. Then we have

$$
\pi_{1}^{-1}([0,0])=\bigcup_{\epsilon \in \Theta_{c p t}(\alpha)} M_{\epsilon}(\alpha) .
$$

Proof. (1) Define a $\mathbb{C}^{\times}$-action on $X(\alpha, 0)$ by $[z, w] s=[s z, s w]$ for $s \in \mathbb{C}^{\times}$and $(z, w) \in \mu_{\mathbb{C}}^{-1}(0)^{\alpha-s s}$. We also define a $\mathbb{C}^{\times}$-action on $X(0,0)$ in a similar way. Then the natural morphism $\pi_{1}:\left(X(\alpha, 0), I_{1}\right) \rightarrow\left(X(0,0), I_{1}\right)$ is $\left(\mathbb{C}^{\times} \times T^{n}\right)$-equivariant. So, if we define a 1-parameter transformation group $\left\{\widetilde{\phi}_{t}\right\}$ of $X(\alpha, 0)$ by $\widetilde{\phi}_{t}([z, w])=$ $\left[e^{-t} z, e^{-t} w\right]$, then the map $\widetilde{\phi}_{t}: X(\alpha, 0) \rightarrow X(\alpha, 0)$ is $T^{n}$-equivariant. Similarly, we can also define a 1-parameter transformation group $\left\{\phi_{t}\right\}$ of $X(0,0)$, which is $T^{n}$-equivariant. Since $[0,0]$ is the unique fixed point in $X(0,0)$ for the $\mathbb{C}^{\times}$-action, we have

$$
\lim _{t \rightarrow \infty} \pi_{1}\left(\widetilde{\phi}_{t}([z, w])\right)=\lim _{t \rightarrow \infty} \phi_{t}\left(\pi_{1}([z, w])\right)=[0,0]
$$

for any $[z, w] \in X(\alpha, 0)$. Thus we see that $\pi_{1}^{-1}([0,0])$ is a $T^{n}$-equivariant deformation retract of $X(\alpha, 0)$.

(2) Suppose that $\pi_{1}([z, w])=[0,0]$, that is,

$$
(0,0) \in \overline{(z, w) K_{\mathbb{C}}}=\overline{\left\{\left(z \zeta, w \zeta^{-1}\right) \mid \zeta \in K_{\mathbb{C}}\right\}} .
$$


Therefore, we have $z_{i} w_{i}=0$ for $i=1, \ldots, N$. We also have $X=\sum_{i=1}^{N} a_{i} X_{i} \in k$ such that $a_{i}>0$ if $z_{i} \neq 0$, and $a_{i}<0$ if $w_{i} \neq 0$. Since $\iota^{*} u_{j} \neq 0$ for any $j=1, \ldots, N$, we may assume that $a_{j} \neq 0$ for any $j=1, \ldots, N$. If we define $\epsilon \in \Theta$ by $\epsilon(i)=1$ if $a_{i}>0$, and $\epsilon(i)=-1$ if $a_{i}<0$, then we have $(z, w) \in V_{\epsilon}$ and $\pi_{1}\left(M_{\epsilon}\right)=[0,0]$. By Lemma 3.5, $M_{\epsilon}(\alpha)$ is compact. So we have $\pi_{1}^{-1}([0,0]) \subset \bigcup_{\epsilon \in \Theta_{c p t}(\alpha)} M_{\epsilon}(\alpha)$. By a similar argument we have $\pi_{1}^{-1}([0,0]) \supset \bigcup_{\epsilon \in \Theta_{\text {cpt }}(\alpha)} M_{\epsilon}(\alpha)$.

Remark 4.2 If $\iota^{*} u_{N}=0$, then we have $K \subset T^{N-1}$ and $X(\alpha, 0)$ is decomposed into the product of a lower dimensional toric hyperkähler variety and $\mathbb{H}$. Therefore the assumption of Proposition 4.1 can be dropped easily if we slightly change the statement. See $[\mathrm{BD}, \mathrm{HS}]$ and the next subsection for detail.

By Proposition 4.1 we call the set $\pi_{1}^{-1}([0,0])$ the core of the toric hyperkähler orbifold $X(\alpha, 0)$. The above proof of (1) is based on the argument in $[\mathrm{Kr}$, $\mathbf{N 1}$, where the similar result is proved in the case of quiver varieties. Bielawski and Dancer proved (1) and (2) in a slightly different formulation [BD].

Proof of Proposition 3.5. Let us prove the surjectivity of the natural morphism $\pi_{1}: X(\alpha, \beta) \rightarrow X(0, \beta)$. Take an arbitrary point $(a, b) \in \mu^{-1}(0, \beta)$. If we set $W=\sum_{i=1}^{N} \mathbb{R}\left|a_{i}\right|^{2} \iota^{*} u_{i}+\sum_{i=1}^{N} \mathbb{R}\left|b_{i}\right|^{2}\left(-\iota^{*} u_{i}\right) \subset k^{*}$, then we have

$$
W=\left\{v \in k^{*} \mid\left\langle v, \underline{k}_{(a, b)}\right\rangle=0\right\},
$$

where $\underline{k}_{(a, b)}=\operatorname{Lie} K_{(a, b)} \subset k$ is the Lie algebra of the isotropy subgroup $K_{(a, b)} \subset K$ at $(a, b)$. Set $J=\left\{i \mid \iota^{*} u_{i} \notin W\right\}$. Since $a_{i}=b_{i}=0$ for any $i \in J$, by Lemma 3.4 we have $0 \in \sum_{i \notin J} \mathbb{R}_{>0}\left|a_{i}\right|^{2} \iota^{*} u_{i}+\sum_{i \notin J} \mathbb{R}_{>0}\left|b_{i}\right|^{2}\left(-\iota^{*} u_{i}\right)$. Therefore we have

$$
W=\sum_{i \notin J} \mathbb{R}_{>0}\left|a_{i}\right|^{2} \iota^{*} u_{i}+\sum_{i \notin J} \mathbb{R}_{>0}\left|b_{i}\right|^{2}\left(-\iota^{*} u_{i}\right)
$$

On the other hand, $\alpha \in k^{*}$ induces $\underline{\alpha} \in\left(\underline{k}_{(a, b)}\right)^{*}$. Let $\underline{X}(\underline{\alpha}, \underline{0})$ and $\underline{X}(\underline{0}, \underline{0})$ be hyperkähler quotients of $\mathbb{H}^{J}=\left\{(z, w) \in \mathbb{H}^{N} \mid z_{i}=w_{i}=0\right.$ for any $\left.i \notin J\right\}$ by $K_{(a, b)}$ at $(\underline{\alpha}, \underline{0}),(\underline{0}, \underline{0}) \in\left(\underline{k}_{(a, b)}\right)^{*} \times\left(\underline{k}_{(a, b) \mathbb{C}}\right)^{*}$ respectively. Let $\underline{\pi}_{1}: \underline{X}(\underline{\alpha}, \underline{0}) \rightarrow \underline{X}(\underline{0}, \underline{0})$ be the natural morphism. By the proof of Proposition 4.1 (2) we can take a point $[c, d] \in\left(\underline{\pi}_{1}\right)^{-1}([0,0])$, where $(c, d) \in \mathbb{H}^{J}$. Namely, we have $(0,0) \in \overline{(c, d) K_{(a, b)}}$. If we set $(z, w)=(a, b)+(c, d) \in \mathbb{H}^{N}$, then we have $(a, b) \in \overline{(z, w) K_{(a, b)}}$. It is obvious that $(z, w) \in \mu_{\mathbb{C}}^{-1}(\beta)$. Moreover, we have

$$
\underline{\alpha} \in \sum_{i \in J} \mathbb{R}_{\geq 0}\left|c_{i}\right|^{2} \underline{\iota}^{*} u_{i}+\sum_{i \in J} \mathbb{R}_{\geq 0}\left|d_{i}\right|^{2}\left(-\underline{\iota}^{*} u_{i}\right),
$$

where $\underline{\iota}: \underline{k}_{(a, b)} \rightarrow t^{J}$ is the natural inclusion and $t^{J}=\operatorname{span}\left\{X_{i} \mid i \in J\right\} \subset t^{N}$. Therefore, by (4.1), (4.2) and (4.3), we have $(z, w) \in \mu_{\mathbb{C}}^{-1}(\beta)^{\alpha-s s}$. Thus we proved $\pi_{1}([z, w])=[a, b]$.

\subsection{The Betti Numbers and the Cohomology Rings}

By Proposition 4.1] to study the topology of a toric hyperkähler orbifold $X(\alpha, 0)$, it is enough to study the topology of its core. To do this, the action of the quotient torus plays an important role. Actually Bielawski and Dancer computed the Betti 
numbers $\mathrm{BD}$, and the author determined the cohomology ring [Ko1 by using it. In this section we review these results.

Fix $(\alpha, 0) \in\left(k^{*} \times k_{\mathbb{C}}^{*}\right)_{r e g}$. First we describe the hyperkähler moment map

$$
\mu_{T^{n}}=\left(\mu_{T^{n}, 1}, \mu_{T^{n} \mathbb{C}}\right): X(\alpha, 0) \rightarrow\left(t^{n}\right)^{*} \otimes \mathbb{R}^{3} \cong\left(t^{n}\right)^{*} \times\left(t_{\mathbb{C}}^{n}\right)^{*}
$$

for the action of the quotient torus $T^{n}=T^{N} / K$ on $X(\alpha, 0)$. Fix $h \in\left(t^{N}\right)^{*}$ such that $\iota^{*} h=\alpha$. Then the hyperkähler moment map $\mu_{T^{n}}: X(\alpha, 0) \rightarrow\left(t^{n}\right)^{*} \otimes \mathbb{R}^{3}$ is given by

$$
\begin{aligned}
& \mu_{T^{n}, 1}([z, w])=\pi \sum_{i=1}^{N}\left(\left|z_{i}\right|^{2}-\left|w_{i}\right|^{2}\right) u_{i}-h \in\left(t^{n}\right)^{*} \subset\left(t^{N}\right)^{*}, \\
& \mu_{T^{n} \mathbb{C}}([z, w])=-2 \pi \sqrt{-1} \sum_{i=1}^{N} z_{i} w_{i} u_{i} \in\left(t_{\mathbb{C}}^{n}\right)^{*} \subset\left(t_{\mathbb{C}}^{N}\right)^{*} .
\end{aligned}
$$

Note that $\mu_{T^{N}, 1}(z, w)-h \in \operatorname{Ker}\left\{\iota^{*}:\left(t^{N}\right)^{*} \rightarrow k^{*}\right\}=\operatorname{Im}\left\{\pi^{*}:\left(t^{n}\right)^{*} \rightarrow\left(t^{N}\right)^{*}\right\}$. Similarly, we have $-2 \pi \sqrt{-1} \sum_{i=1}^{N} z_{i} w_{i} u_{i} \in \operatorname{Ker} \iota^{*} \otimes \mathbb{C}=\operatorname{Im} \pi^{*} \otimes \mathbb{C}$.

We define a hyperplane $F_{i} \subset\left(t^{n}\right)^{*}$ by

$$
F_{i}=\left\{p \in\left(t^{n}\right)^{*} \mid\left\langle\pi^{*} p+h, X_{i}\right\rangle=0\right\} \quad \text { for } i=1, \ldots, N .
$$

We note that $F_{i}=\emptyset$ if $\pi\left(X_{i}\right)=0$, because we assume $(\alpha, 0) \in\left(k^{*} \times k_{\mathbb{C}}^{*}\right)_{\text {reg }}$. Let $\Theta$ be the set of all maps from $\{1, \ldots, N\}$ to $\{1,-1\}$ as in the last section. Then these hyperplanes divide $\left(t^{n}\right)^{*}$ into a finite number of polyhedra $\left\{\Delta_{\epsilon} \mid \epsilon \in \Theta\right\}$, where $\Delta_{\epsilon} \in\left(t^{n}\right)^{*}$ is defined by

$$
\Delta_{\epsilon}=\left\{p \in\left(t^{n}\right)^{*} \mid \epsilon(i)\left\langle\pi^{*} p+h, X_{i}\right\rangle \geq 0 \text { for any } i=1, \ldots, N\right\} .
$$

We note that some $\Delta_{\epsilon}$ may be empty. Then we have the following lemma.

Lemma $4.3[z, w] \in \mu_{T^{n}}^{-1}\left(\Delta_{\epsilon}, 0\right)$ if and only if $(z, w) \in V_{\epsilon} \cap \mu_{1}^{-1}(\alpha)$. Namely, $M_{\epsilon}(\alpha)=\mu_{T^{n}}^{-1}\left(\Delta_{\epsilon}, 0\right)$ holds.

Proof. First we note that $[z, w] \in \mu_{T^{n}}^{-1}\left(\left(t^{n}\right)^{*}, 0\right)$ if and only if $z_{i} w_{i}=0$ for all $i=1, \ldots, N$. If we set $p=\mu_{T^{n}, 1}([z, w])$, we have

$$
\left\langle\pi^{*} p+h, X_{i}\right\rangle=\pi\left(\left|z_{i}\right|^{2}-\left|w_{i}\right|^{2}\right) \quad \text { for } i=1, \ldots, N .
$$

Then the lemma follows.

We want to study the topology of the core. Note that each component of the core is an ordinary toric orbifold, even if we do not assume that $\iota^{*} u_{i} \neq 0$ for any $i=1, \ldots, N$. So we have to investigate how these components intersect to each other. This is described by the polyhedral complex, which is define by

$$
\mathcal{C}(X(\alpha, 0))=\left\{\sigma \mid \sigma \text { is a compact face of a polyhedron } \Delta_{\epsilon} \text { for some } \epsilon \in \Theta\right\} .
$$

It should be remarked that, to define $\mathcal{C}(X(\alpha, 0))$, we need $h \in\left(t^{N}\right)^{*}$ such that $\iota^{*} h=\alpha$. However, $\mathcal{C}(X(\alpha, 0))$ is determined by $\alpha$ up to parallel translation. So we use this notation.

Note that the polyhedral complex $\mathcal{C}(X(\alpha, 0))$ is determined by the hyperplanes $F_{1}, \ldots, F_{N}$. Therefore, the cohomology of the toric hyperkähler orbifold $X(\alpha, 0)$ is described in terms of the hyperplanes $F_{1}, \ldots, F_{N}$ or the polyhedral complex $\mathcal{C}(X(\alpha, 0))$. In fact, we have the following. 
Theorem 4.4 $\mathrm{BD}$ Fix $(\alpha, 0) \in\left(k^{*} \times k_{\mathbb{C}}^{*}\right)_{\text {reg. }}$. Let $d_{k}$ be the number of $k$-simplexes of the polyhedral complex $\mathcal{C}(X(\alpha, 0))$. Then the Poincaré polynomial $P_{t}(X(\alpha, 0))$ is given by

$$
P_{t}(X(\alpha, 0))=\sum_{k=0}^{n} d_{k}\left(t^{2}-1\right)^{k} .
$$

Note that the Poincaré polynomial of an ordinary toric orbifold is the same form if we replace $\mathcal{C}(X(\alpha, 0))$ by the convex polytope associated to the toric orbifold. See Section 4.5 in Ful.

In Proposition 3.7 we stated the condition so that $X(\alpha, \beta)$ is a smooth manifold for $(\alpha, \beta) \in\left(k^{*} \times k_{\mathbb{C}}^{*}\right)_{\text {reg }}$. Recall the condition is the one for the subtorus $K$, and independent of $(\alpha, \beta)$. Under the condition $\mu^{-1}(\alpha, \beta)$ can be viewed as a principal $K$-bundle on $X(\alpha, \beta)$. Let $L_{i}$ be a line bundle on $X(\alpha, \beta)$ associated the character $\iota^{*} u_{i}$ of $K$ for $i=1, \ldots, N$.

Lemma 4.5 Suppose that $\Delta_{\epsilon} \cap F_{i}$ is a face of $\Delta_{\epsilon}$ of codimension one. Then the homology class represented by $\mu_{T^{n}}^{-1}\left(\Delta_{\epsilon} \cap F_{i}, 0\right)$ is the Poincaré dual of $\epsilon(i) c_{1}\left(L_{i}\right)$ in $M_{\epsilon}(\alpha)$.

Proof. Suppose $[z, w] \in \mu_{T^{n}}^{-1}\left(\left(t^{n}\right)^{*}, 0\right)$. Then $[z, w] \in F_{i}$ if and only if $z_{i}=w_{i}=0$. Then we have

$$
\mu_{T^{n}}^{-1}\left(\Delta_{\epsilon} \cap F_{i}, 0\right)=\left\{\begin{array}{l}
\left\{[z, w] \in M_{\epsilon}(\alpha) \mid z_{i}=0\right\} \quad \text { if } \epsilon(i)=1, \\
\left\{[z, w] \in M_{\epsilon}(\alpha) \mid w_{i}=0\right\} \quad \text { if } \epsilon(i)=-1 .
\end{array}\right.
$$

Note that the divisor defined by the equation $z_{i}=0$ in $\left(X(\alpha, \beta), I_{1}\right)$ corresponds to $L_{i}$ and that the divisor defined by the equation $w_{i}=0$ in $\left(X(\alpha, \beta), I_{1}\right)$ corresponds to $L_{i}^{*}$. So we finish the proof.

The cohomology ring of a toric hyperkähler manifold $X(\alpha, \beta)$ is described as follows.

Theorem 4.6 Ko1 Suppose that $(\alpha, \beta) \in\left(k^{*} \times k_{\mathbb{C}}^{*}\right)_{r e g}$ and $X(\alpha, \beta)$ is a smooth manifold. Define a ring homomorphism $\Phi: \mathbb{Z}\left[u_{1}, \ldots, u_{N}\right] \rightarrow H^{*}(X(\alpha, \beta) ; \mathbb{Z})$ by $\Phi\left(u_{i}\right)=c_{1}\left(L_{i}\right)$. Then the following holds:

(1) The map $\Phi$ is surjective. So we have a ring isomorphism

$$
H^{*}(X(\alpha, \beta) ; \mathbb{Z}) \cong \mathbb{Z}\left[u_{1}, \ldots, u_{N}\right] / \operatorname{ker} \Phi .
$$

(2) The ideal $\operatorname{ker} \Phi$ is generated by the following two types of elements:

(i) (linear relations) $\sum_{i=1}^{N} a_{i} u_{i} \in \operatorname{ker} \iota^{*} \cap\left(t_{\mathbb{Z}}^{N}\right)^{*}$,

(ii) (nonlinear relations) $\prod_{i \in J} u_{i}$ for all $J \subset\{1, \ldots, N\}$ such that $\bigcap_{i \in J} F_{i}=\emptyset$.

Remark 4.7 For $\epsilon \in \Theta_{\text {cpt }}(\alpha)$ we have $H\left(M_{\epsilon}(\alpha), \mathbb{Z}\right) \cong \mathbb{Z}\left[u_{1}, \ldots, u_{N}\right] / I_{\epsilon}$, where the ideal $I_{\epsilon}$ are generated by the following two types of elements;

(a) the same as $(i)$ in Theorem 4.6 (2),

(b) $\prod_{i \in J} u_{i} \quad$ for all $J \subset\{1, \ldots, N\}$ such that $\left(\bigcap_{i \in J} F_{i}\right) \cap \Delta_{\epsilon}=\emptyset$.

To compute the cohomology of the core, we have to take all $M_{\epsilon}(\alpha)$ for $\epsilon \in \Theta_{\text {cpt }}(\alpha)$ into account at same time. This is the geometric meaning of the nonlinear relations in Theorem 4.6. 
Remark 4.8 In HS Hausel and Sturmfels generalized Theorem 4.6 to orbifold cases, where the coefficient $\mathbb{Z}$ is replaced by $\mathbb{R}$. In Subsection 4.4 we discuss their proof. Our proof, which will be described in the next subsection, also works for orbifold cases if $L_{i}$ is considered as an orbi-line bundle on $X(\alpha, \beta)$.

\subsection{Outline of the proofs of Theorems 4.4 and 4.6}

Let us sketch the idea of the proofs of Theorems 4.4 and 4.6. By Proposition 3.6 it is enough to compute the cohomology ring of $X(\alpha, 0)$ for $(\alpha, 0) \in\left(k^{*} \times k_{\mathbb{C}}^{*}\right)_{\text {reg }}$. Let $F_{1}, \ldots, F_{N}$ be the hyperplanes associated to $X(\alpha, 0)$. Let $Y_{1}$ and $\mathcal{C}\left(Y_{1}\right)$ be another toric hyperkähler variety and the polyhedral complex associated to the hyperplanes $F_{1}, \ldots, F_{N-1}$ respectively. By Proposition 3.6, the topology of $X(\alpha, 0)$ does not change as far as $(\alpha, 0) \in\left(k^{*} \times k_{\mathbb{C}}^{*}\right)_{r e g}$. Since the variation of $\alpha$ corresponds to parallel translations of the hyperplanes, we can choose $\alpha$ so that $\mathcal{C}\left(Y_{1}\right)$ is contained in one of the components of $k \backslash F_{N}$. Then we consider another toric hyperkähler variety $Y_{2}$ associated to the hyperplanes $F_{1} \cap F_{N}, \ldots, F_{N-1} \cap F_{N}$. Bielawski and Dancer expressed the Betti numbers of $X(\alpha, 0)$ in terms of those of $Y_{1}$ and $Y_{2}$ by the Meyer-Vietoris argument. Then by an inductive argument they computed the Betti numbers of $X(\alpha, 0)$ [BD]. In [Ko1 the author determined the cohomology ring of $X(\alpha, 0)$ from the one of $Y_{1}$ and $Y_{2}$ by a refinement of the above argument.

\subsection{Another proof}

Another proof was given by Hausel and Sturmfels. In this subsection we explain their proof. Let us consider a symplectic quotient $L(\alpha)=\mu_{1}^{-1}(\alpha) / K$ of $\mathbb{H}^{N}$ by $K$, which is a non-compact ordinary toric variety. Note that a toric hyperkähler variety $\left(X(\alpha, \beta), I_{1}\right)=\left(\mu_{1}^{-1}(\alpha) \cap \mu_{\mathbb{C}}^{-1}(\beta)\right) / K$ is a subvariety of $L(\alpha)$. Hausel and Sturmfels defined the core of $L(\alpha)$, which is a deformation retract of $L(\alpha)$ for $(\alpha, 0) \in\left(k^{*} \times k_{\mathbb{C}}^{*}\right)_{\text {reg }}$. Moreover, they observed that the core of $X(\alpha, 0)$ is the same as the one of $L(\alpha)$. So they proved that the cohomology ring of $X(\alpha, 0)$ is isomorphic to the one of $L(\alpha)$, which can be computed by the standard argument for ordinary toric varieties [Ful]. The argument can be considered as a refinement of the Morse theory. So the properness of a Morse function is important. Since $L(\alpha)$ is non-compact, Hausel and Sturmfels carefully choose an appropriate Morse function and computed the cohomology ring of $X(\alpha, 0)[\mathrm{HS}]$.

Here we prove that $X(\alpha, 0)$ is homotopy equivalent to $L(\alpha)$ in a different way from them.

Proposition 4.9 Suppose $(\alpha, 0) \in\left(k^{*} \times k_{\mathbb{C}}^{*}\right)_{\text {reg. }}$. Then $L(\alpha)$ is diffeomorphic to the product $X(\alpha, 0) \times k_{\mathbb{C}}^{*}$. In particular, $X(\alpha, 0)$ is homotopy equivalent to $L(\alpha)$.

Proof. Since $(\alpha, 0) \in\left(k^{*} \times k_{\mathbb{C}}^{*}\right)_{\text {reg }}$, due to Lemma 3.6. the natural morphism $\pi_{2}:\left(X\left(\alpha, \alpha_{2}, \alpha_{3}\right), I_{2}\right) \rightarrow\left(X\left(\alpha, 0, \alpha_{3}\right), I_{2}\right)$ is a biholomorphic map for any $\alpha_{2}, \alpha_{3} \in$ $k^{*}$. Similarly, $\pi_{3}:\left(X\left(\alpha, 0, \alpha_{3}\right), I_{3}\right) \rightarrow\left(X(\alpha, 0,0), I_{3}\right)$ is also a biholomorphic map. So we have a diffeomorphism $\pi_{3} \circ \pi_{2}: X(\alpha, \beta) \rightarrow X(\alpha, 0)$ for any $\beta \in k_{\mathbb{C}}^{*}$. Since $L(\alpha)$ is equal to $\bigcup_{\beta \in k_{\mathrm{C}}^{*}} X(\alpha, \beta)$, this map induces a diffeomorphism from $L(\alpha)$ to $X(\alpha, 0) \times k_{\mathbb{C}}^{*}$.

We refer the reader to $[\mathrm{P}$ for other methods to compute the cohomology ring and also for the computation of the equivariant cohomology of $X(\alpha, \beta)$ for the torus action. 


\subsection{Another presentation of the cohomology ring}

The topology of a toric hyperkähler variety $X(\alpha, \beta)$ for $(\alpha, \beta) \in\left(k^{*} \times k_{\mathbb{C}}^{*}\right)_{r e g}$ is determined only by the subtorus $K$. So we have a presentation of its cohomology ring in terms of $K$ as follows.

Theorem 4.10 Suppose the same assumption as in Theorem 4.6 . Let $\Lambda^{(1)}=$ $\left\{H_{1}^{(1)}, \ldots, H_{l}^{(1)}\right\}$ be the set of 1-dimensional isotropy subgroups in $K$. Fix a nonzero element $Y_{s} \in$ LieH $_{s}^{(1)}$ and set $J_{s}=\left\{i \in\{1, \ldots, N\} \mid\left\langle u_{i}, Y_{s}\right\rangle \neq 0\right\}$ for $s=1, \ldots, l$. Then (ii) (nonlinear relations) in Theorem 4.6 (2) can be replaced by the following;

$(i i)^{\prime}$ (nonlinear relations) $\prod_{i \in J_{s}} u_{i}$ for $s=1, \ldots, l$.

Since the above presentation is slightly different from the one in [Ko1, we give a proof here, assuming Theorem 4.6.

Proof. Let $I$ and $I^{\prime}$ be the ideals in $\mathbb{Z}\left[u_{1}, \ldots, u_{N}\right]$ in Theorem 4.6 and Theorem 4.10 respectively. First we show $I^{\prime} \subset I$. We have to show $\bigcap_{i \in J_{s}} F_{i}=\emptyset$ for any $s=1, \ldots, l$. Suppose that there exists $p \in \bigcap_{i \in J_{s}} F_{i}$, that is, $\left\langle\pi^{*} p+h, X_{i}\right\rangle=0$ for all $i \in J_{s}$. Therefore we have $\pi^{*} p+h \in \operatorname{span}\left\{u_{i} \mid i \notin J_{s}\right\}$. Thus we have $\alpha \in \operatorname{span}\left\{\iota^{*} u_{i} \mid i \notin J_{s}\right\}$. Since $(\alpha, 0) \in\left(k^{*} \times k_{\mathbb{C}}^{*}\right)_{\text {reg }}$, according to Lemma 3.6, we have $\operatorname{span}\left\{\iota^{*} u_{i} \mid i \notin J_{s}\right\}=k^{*}$. Since $\left\langle Y_{s}, \iota^{*} u_{i}\right\rangle=0$ for all $i \notin J_{s}$, we have $Y_{s}=0$. This is a contradiction. Thus we see that $\bigcap_{i \in J_{s}} F_{i}=\emptyset$.

Next we show $I \subset I^{\prime}$. Suppose that $\bigcap_{i \in J} F_{i}=\emptyset$. We have to prove that there exists $s \in\{1, \ldots, l\}$ such that $J_{s} \subset J$. First we prove that $\operatorname{span}\left\{\iota^{*} u_{i} \mid i \notin J\right\} \varsubsetneqq k^{*}$. Suppose that $\operatorname{span}\left\{\iota^{*} u_{i} \mid i \notin J\right\}=k^{*}$. Then we can choose $A \subset J^{c}$, where $J^{c}=\{1, \ldots, N\} \backslash J$, such that $\left\{\iota^{*} u_{i} \mid i \in A\right\}$ forms a basis of $k^{*}$. So we can write $\alpha=\sum_{i \in A} c_{i} \iota^{*} u_{i}$. Therefore there exists $(z, w) \in \mu^{-1}(\alpha, 0)$ such that $z_{i}=w_{i}=0$ if $i \notin A, w_{i}=0$ if $i \in A$ and $c_{i} \geq 0, z_{i}=0$ if $i \in A$ and $c_{i} \leq 0$. If we set $p=\mu_{T^{n}, 1}([z, w])$, according to Lemma 4.5, we have $p \in \bigcap_{i \in A^{c}} F_{i} \subset \bigcap_{i \in J} F_{i}$. This is a contradiction. Thus we proved $\operatorname{span}\left\{\iota^{*} u_{i} \mid i \notin J\right\} \varsubsetneqq k^{*}$. So there exists a wall $W_{s}$ such that $\operatorname{span}\left\{\iota^{*} u_{i} \mid i \notin J\right\} \subset W_{s}$. This implies $J_{s} \subset J$.

\subsection{The hyperkähler Kirwan map}

Assume that a toric hyperkähler variety $X(\alpha, \beta)$ is smooth. Let $H_{K}^{*}\left(\mathbb{H}^{N} ; \mathbb{Z}\right)$ be the $K$-equivariant cohomology of $\mathbb{H}^{N}$. Define the map

$$
\kappa_{\mathbb{Z}}: H_{K}^{*}\left(\mathbb{H}^{N} ; \mathbb{Z}\right) \rightarrow H^{*}(X(\alpha, \beta) ; \mathbb{Z})
$$

to be the composition of the restriction map $r: H_{K}^{*}\left(\mathbb{H}^{N} ; \mathbb{Z}\right) \rightarrow H_{K}^{*}\left(\mu^{-1}(\alpha, \beta) ; \mathbb{Z}\right)$ and the natural isomorphism $i: H_{K}^{*}\left(\mu^{-1}(\alpha, \beta) ; \mathbb{Z}\right) \rightarrow H^{*}(X(\alpha, \beta) ; \mathbb{Z})$. The map $\kappa_{\mathbb{Z}}$ is an hyperkähler analogue of the Kirwan map for a symplectic quotient. Kirwan proved that the Kirwan map is surjective for symplectic quotients in $\mathbb{R}$-coefficients (even if the quotient is smooth) under very weak assumptions. Theorem 4.6 implies that the hyperkähler Kirwan map is surjective for toric hyperkähler manifolds even in $\mathbb{Z}$-coefficients, because $H_{K}^{*}\left(\mathbb{H}^{N} ; \mathbb{Z}\right) \cong S^{*}\left(k_{\mathbb{Z}}^{*}\right) \cong \mathbb{Z}\left[u_{1}, \ldots, u_{N}\right] /\left(\operatorname{ker} \iota^{*} \cap\left(t_{\mathbb{Z}}^{N}\right)^{*}\right)$, where $S^{*}\left(k_{\mathbb{Z}}^{*}\right)$ is the symmetric power of $k_{\mathbb{Z}}^{*}$. In the orbifold case the hyperkähler Kirwan map

$$
\kappa: H_{K}^{*}\left(\mathbb{H}^{N} ; \mathbb{R}\right) \rightarrow H^{*}(X(\alpha, \beta) ; \mathbb{R})
$$


is also surjective. In Ko2 the author computed the cohomology ring and proved the surjectivity of the hyperkähler Kirwan map for the hyperkähler polygon spaces, which are hyperkähler quotients of $\mathbb{H}^{N}$ by certain non-abelian Lie groups. It is not known whether the hyperkähler Kirwan map is surjective or not in general. Surjectivity of the hyperkähler Kirwan map is one of the central topics for study of the topology of hyperkähler quotients.

\section{$5 \quad$ Examples}

In this section we give two examples, which illustrate the results in other sections. The first one is the most fundamental.

Example 5.1 Consider the subtorus $K$ of $T^{n+1}$ whose Lie algebra $k$ is spanned by $X_{1}+\cdots+X_{n+1}$. If we denote the dual basis by $v \in k^{*}$, then we have $\iota^{*} u_{1}=$ $\cdots=\iota^{*} u_{n+1}=v$.

The hyperkähler moment map for the action of $K$ on $\mathbb{H}^{n+1}$

$$
\mu=\left(\mu_{1}, \mu_{\mathbb{C}}\right): \mathbb{H}^{n+1} \rightarrow k^{*} \otimes \mathbb{R}^{3} \cong k^{*} \times k_{\mathbb{C}}^{*}
$$

is given by

$$
\begin{aligned}
& \mu_{1}(z, w)=\pi \sum_{i=1}^{n+1}\left(\left|z_{i}\right|^{2}-\left|w_{i}\right|^{2}\right) v \in k^{*}, \\
& \mu_{\mathbb{C}}(z, w)=-2 \pi \sqrt{-1}\left(\sum_{i=1}^{n+1} z_{i} w_{i}\right) v \in k_{\mathbb{C}}^{*} .
\end{aligned}
$$

Then we have $\left(k^{*} \times k_{\mathbb{C}}^{*}\right)_{r e g}=k^{*} \times k_{\mathbb{C}}^{*} \backslash\{(0,0)\}$.

First we consider the case $\beta=0$. Fix $\alpha_{+}=a_{+} v$ and $\alpha_{-}=a_{-} v$, where $a_{+}>0, a_{-}<0$. By Lemma 3.4 we have

$$
\mu_{\mathbb{C}}^{-1}(0)^{\alpha_{+}-s s}=\left\{(z, w) \in \mu_{\mathbb{C}}^{-1}(0) \mid z \neq 0\right\} .
$$

By Proposition 3.6 we have $\left(X\left(\alpha_{+}, 0\right), I_{1}\right)=\mu_{\mathbb{C}}^{-1}(0)^{\alpha_{+}-s s} / K_{\mathbb{C}}$, which is biholomorphic to the total space of the cotangent bundle of $\mathbb{C} P^{n}$. Similarly, we have

$$
\mu_{\mathbb{C}}^{-1}(0)^{\alpha_{-}-s s}=\left\{(z, w) \in \mu_{\mathbb{C}}^{-1}(0) \mid w \neq 0\right\} .
$$

Therefore we have $\left(X\left(\alpha_{-}, 0\right), I_{1}\right)=\mu_{\mathbb{C}}^{-1}(0)^{\alpha_{-}-s s} / K_{\mathbb{C}}$, which is also biholomorphic to $T^{*} \mathbb{C} P^{n}$.

Next we consider the case $\beta \neq 0$. Fix an arbitrary $\alpha$. Then, by Proposition 3.6. $\left(X(\alpha, \beta), I_{1}\right) \cong\left(X(0, \beta), I_{1}\right)$ is an affine variety. Therefore $\left(X(\alpha, \beta), I_{1}\right)$ is diffeomorphic to $T^{*} \mathbb{C} P^{n}$, but not biholomorphic as a complex manifold.

By Theorems 4.6 and 4.10 we have $H^{*}(X(\alpha, \beta), \mathbb{Z}) \cong \mathbb{Z}\left[u_{1}, \ldots, u_{n+1}\right] / I$, where the ideal $I$ is generated by two types of elements. The linear relations are generated by $u_{1}-u_{2}, u_{2}-u_{3}, \ldots u_{n}-u_{n+1}$. The nonlinear relation is generated by $u_{1} u_{2} \ldots u_{n+1}$. Therefore we have

$$
\begin{aligned}
& H^{*}(X(\alpha, \beta), \mathbb{Z}) \\
& \cong \mathbb{Z}\left[u_{1}, \ldots, n+1\right] /\left(u_{1}-u_{2}, u_{2}-u_{3}, \ldots u_{n}-u_{n+1}, u_{1} u_{2} \ldots u_{n+1}\right) \\
& \cong \mathbb{Z}[v] /\left(v^{n+1}\right) .
\end{aligned}
$$


Example 5.2 Consider the subtorus $K$ of $T^{5}$ whose Lie algebra $k$ is spanned by $X_{1}+X_{2}+X_{4}$ and $X_{1}+X_{3}+X_{5}$. Let $\left\{v_{1}, v_{2}\right\}$ be the dual basis. Then we have $\iota^{*} u_{1}=v_{1}+v_{2}, \iota^{*} u_{2}=\iota^{*} u_{4}=v_{1}$ and $\iota^{*} u_{3}=\iota^{*} u_{5}=v_{2}$.

The hyperkähler moment map for the action of $K$ on $\mathbb{H}^{5}$

$$
\mu=\left(\mu_{1}, \mu_{\mathbb{C}}\right): \mathbb{H}^{5} \rightarrow k^{*} \otimes \mathbb{R}^{3} \cong k^{*} \times k_{\mathbb{C}}^{*}
$$

is given by

$$
\begin{aligned}
& \mu_{1}(z, w)=\pi \sum_{i=1,2,4}\left(\left|z_{i}\right|^{2}-\left|w_{i}\right|^{2}\right) v_{1}+\pi \sum_{i=1,3,5}\left(\left|z_{i}\right|^{2}-\left|w_{i}\right|^{2}\right) v_{2} \in k^{*}, \\
& \mu_{\mathbb{C}}(z, w)=-2 \pi \sqrt{-1}\left(\sum_{i=1,2,4} z_{i} w_{i}\right) v_{1}-2 \pi \sqrt{-1}\left(\sum_{i=1,3,5} z_{i} w_{i}\right) v_{2} \in k_{\mathbb{C}}^{*} .
\end{aligned}
$$

We have three 1-dimensional isotropy subgroups $H_{1}^{(1)}, H_{1}^{(2)}, H_{1}^{(3)}$, whose Lie algebras are spanned by $Y_{1}=X_{1}+X_{3}+X_{5}, Y_{2}=X_{1}+X_{2}+X_{4}$ and $Y_{3}=$ $X_{2}-X_{3}+X_{4}-X_{5}$ respectively. So we have three walls $W_{1}=\mathbb{R} v_{1}, W_{2}=\mathbb{R} v_{2}$ and $W_{3}=\mathbb{R}\left(v_{1}+v_{2}\right)$ in $k^{*}$. By Proposition 3.6, we see that $(\alpha, 0) \in\left(k^{*} \times k_{\mathbb{C}}^{*}\right)_{\text {reg }}$ if and only if $\alpha \in k^{*} \backslash \bigcup_{i=1,2,3} W_{i}$. Connected components of this set are called chambers. So we have six chambers $\mathcal{C}_{1}, \ldots, \mathcal{C}_{6}$ in $k^{*}$ as in Figure 1 . (This is the chamber structure for $\beta=0$. See Section 6 for the precise definition.)

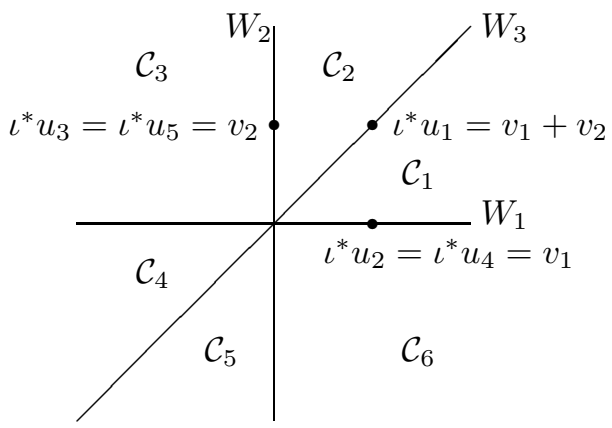

Figure 1

If we take $\alpha_{1}=s v_{1}+t v_{2} \in \mathcal{C}_{1}$, then we have $s>t>0$. Moreover, we have $\Theta_{\text {cpt }}\left(\alpha_{1}\right)=\left\{\epsilon_{1}, \epsilon_{2}\right\}$, where

$$
\epsilon_{1}(i)=1 \text { for } i=1,2,3,4,5, \quad \epsilon_{2}(i)= \begin{cases}1 & \text { for } i=1,2,4 \\ -1 & \text { for } i=3,5\end{cases}
$$

The associated polyhedral complex $\mathcal{C}\left(X\left(\alpha_{1}, 0\right)\right)$ consists of all faces of $\Delta_{\epsilon_{1}}$ and $\Delta_{\epsilon_{2}}$ as in Figure 2, where we take an appropriate coordinate $\left(a_{1}, a_{2}, a_{3}\right)$ in $\left(t^{3}\right)^{*}$ such that $F_{i}=\left\{\left(a_{1}, a_{2}, a_{3}\right) \mid a_{i}=0\right\}$ for $i=1,2,3, F_{4}=\left\{\left(a_{1}, a_{2}, a_{3}\right) \mid a_{1}+a_{2}=s\right\}$ and $F_{5}=\left\{\left(a_{1}, a_{2}, a_{3}\right) \mid a_{1}+a_{3}=t\right\}$. Note that $F_{1} \cap F_{2} \cap F_{4}=F_{1} \cap F_{3} \cap F_{5}=$ $F_{2} \cap F_{3} \cap F_{4} \cap F_{5}=\emptyset$.

Let us compute the cohomology ring of $X\left(\alpha_{1}, 0\right)$. By Theorems 4.6 and 4.10 we have $H^{*}\left(X\left(\alpha_{1}, 0\right), \mathbb{Z}\right) \cong \mathbb{Z}\left[u_{1}, \ldots, u_{5}\right] / I$, where the ideal $I$ is generated by two types of elements. The linear relations are generated by $u_{2}-u_{4}, u_{3}-u_{5}$ and $u_{1}-u_{2}-u_{3}$. The nonlinear relations are generated by $u_{1} u_{2} u_{4}, u_{1} u_{3} u_{5}$ and $u_{2} u_{3} u_{4} u_{5}$. Therefore we have

$$
\begin{aligned}
& H^{*}\left(X\left(\alpha_{1}, 0\right), \mathbb{Z}\right) \\
& \cong \mathbb{Z}\left[u_{1}, \ldots, u_{5}\right] /\left(u_{2}-u_{4}, u_{3}-u_{5}, u_{1}-u_{2}-u_{3}, u_{1} u_{2} u_{4}, u_{1} u_{3} u_{5}, u_{2} u_{3} u_{4} u_{5}\right) \\
& \cong \mathbb{Z}\left[v_{1}, v_{2}\right] /\left(\left(v_{1}+v_{2}\right) v_{1}^{2},\left(v_{1}+v_{2}\right) v_{2}^{2}, v_{1}^{2} v_{2}^{2}\right) .
\end{aligned}
$$




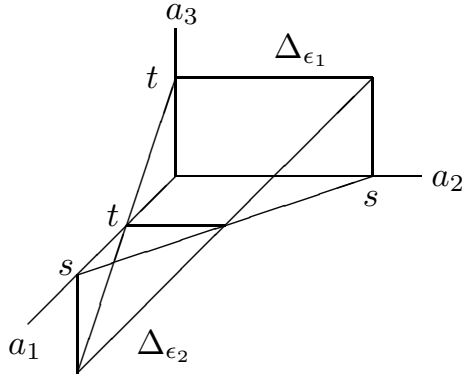

Figure 2

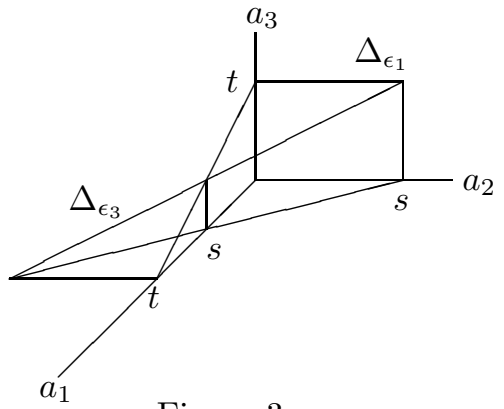

Figure 3

If we take $\alpha_{2}=s v_{1}+t v_{2} \in \mathcal{C}_{2}$, then we have $t>s>0$. Moreover, we have $\Theta_{\text {cpt }}\left(\alpha_{2}\right)=\left\{\epsilon_{1}, \epsilon_{3}\right\}$, where

$$
\epsilon_{1}(i)=1 \text { for } i=1,2,3,4,5, \quad \epsilon_{3}(i)= \begin{cases}1 & \text { for } i=1,3,5 \\ -1 & \text { for } i=2,4\end{cases}
$$

The associated polyhedral complex $\mathcal{C}\left(X\left(\alpha_{2}, 0\right)\right)$ consists of all faces of $\Delta_{\epsilon_{1}}$ and $\Delta_{\epsilon_{3}}$ as in Figure 3.

Since $\mathcal{C}\left(X\left(\alpha_{1}, 0\right)\right)$ and $\mathcal{C}\left(X\left(\alpha_{2}, 0\right)\right)$ have different combinatorial structures, the topology of the core changes. However, by Proposition 3.6, $X\left(\alpha_{1}, 0\right)$ and $X\left(\alpha_{2}, 0\right)$ are diffeomorphic. Moreover the core is a deformation retract of the toric hyperkähler manifold. Therefore we see that the cohomology ring of the core does not change, even if its topology changes. This is an interesting phenomenon.

The variation of $\alpha$ corresponds to parallel translations of the hyperplanes $F_{1}, \ldots F_{N}$. On the other hand, according to Theorem 4.6, the cohomology ring of the core depends only on the subsets $\left\{J \subset\{1, \ldots, N\} \mid \bigcap_{i \in J} F_{i}=\emptyset\right\}$. These subsets are preserved under parallel translations of the hyperplanes, even if the combinatorial structure of the associated polyhedral complex changes. This fact corresponds to the phenomenon mentioned above.

\section{$6 \quad$ Variation of hyperkähler structures}

In [Ko3, the author described how a toric hyperkähler variety $X(\alpha, \beta)$ changes according to the variation of the parameter $(\alpha, \beta)$. First we can explain the geometric meaning of the parameter as follows.

Proposition 6.1 Suppose that the subtorus $K$ satisfies the condition that $X_{i} \notin k$ for $i=1, \ldots, N$. Then the following holds:

(1) The hyperkähler Kirwan map $\kappa: H_{K}^{*}\left(\mathbb{H}^{N} ; \mathbb{R}\right) \rightarrow H^{*}(X(\alpha, \beta) ; \mathbb{R})$ induces an isomorphism $k^{*} \cong H^{2}(X(\alpha, \beta) ; \mathbb{R})$.

(2) The parameter $(\alpha, \beta)$ corresponds to the period of the hyperkähler structure of $X(\alpha, \beta)$. That is, the following holds:

$$
\begin{aligned}
& \kappa(\alpha)=\left[\omega_{1}\right] \in H^{2}(X(\alpha, \beta) ; \mathbb{R}), \\
& \kappa(\beta)=\left[\omega_{\mathbb{C}}\right] \in H^{2}(X(\alpha, \beta) ; \mathbb{C}) .
\end{aligned}
$$

See [Ko3] for the proof. (1) is just a corollary of Theorem 4.6.

Fix $\beta \in k_{\mathbb{C}}^{*}$. Let us describe how the toric hyperkähler variety $X(\alpha, \beta)$ changes according to the variation of $\alpha$. The set $\left(k^{*} \times\{\beta\}\right) \cap\left(k^{*} \times k_{\mathbb{C}}^{*}\right)_{r e g}$ is homeomorphic to $k^{*} \backslash \bigcup_{s \in S_{\beta}} W_{s}$, where $S_{\beta}=\left\{s \in\{1, \ldots, l\} \mid \beta \in W_{s} \otimes \mathbb{C}\right\}$. A connected component 
of the set $k^{*} \backslash \bigcup_{s \in S_{\beta}} W_{s}$ is called a chamber. Note that the chamber structure depends on $\beta \in k_{\mathbb{C}}^{*}$. See Example 5.2 for a concrete example.

Proposition 6.2 Let $k^{*} \backslash \bigcup_{s \in S_{\beta}} W_{s}$ be the chamber structure for a fixed $\beta \in k_{\mathbb{C}}^{*}$. Suppose that $\alpha$ and $\alpha^{\prime}$ belong to the same chamber. Then $\left(X\left(\alpha^{\prime}, \beta\right), I_{1}\right)$ is canonically biholomorphic to $\left(X(\alpha, \beta), I_{1}\right)$.

Proof. By Lemma 3.4, we have $\mu_{\mathbb{C}}^{-1}(\beta)^{\alpha-s s}=\mu_{\mathbb{C}}^{-1}(\beta)^{\alpha^{\prime}-s s}$. So we finish the proof.

Next we discuss the relation between two complex structures corresponding to different chambers. Namely, we study the so called wall-crossing phenomena.

To do this, let us recall a special bimeromorphic map between complex symplectic manifolds, which was discovered by Mukai [M]. Let $X_{+}$be a complex symplectic manifold of complex dimension $2 n$, containing a complex submanifold $V_{+}$biholomorphic to $\mathbb{C} P^{n}$. Since $V_{+}$is a complex Lagrangian submanifold, its normal bundle is biholomorphic to the cotangent bundle $T^{*} V_{+}$. Let $\pi: \widehat{X_{+}} \rightarrow X_{+}$be the blowing-up along $V_{+}$. Then the exceptional divisor $\pi^{-1}\left(V_{+}\right)$is biholomorphic to the projective cotangent bundle $\mathbb{P}\left(T^{*} V_{+}\right)$. This can be viewed as the variety of pairs $(p, h)$ such that $p \in V_{+}, h \in V_{-}$with $p \subset h$, where $V_{-}$is the space of hyperplanes in $V_{+}$. Therefore we can blow-down $\mathbb{P}\left(T^{*} V_{+}\right)$to $V_{-}$and get a new complex symplectic manifold $X_{-}$and the bimeromorphic map $f: X_{+} \rightarrow X_{-}$, which is called the Mukai flop along $V_{+}$. This construction can be generalized directly to the case where $V_{+}$is a $\mathbb{C} P^{r}$-bundle.

Example 6.3 This is a continuation of Example 5.1. Suppose that $\beta=0$. Let $\pi_{ \pm}: X\left(\alpha_{ \pm}, 0\right) \rightarrow X(0,0)$ be the natural morphism. Then $V_{0}=\{[0,0]\}$ is a set of singular points in $X(0,0)$. Note that $(0,0) \in \mathbb{H}^{n+1}$ is the unique fixed point for the action of $K_{\mathbb{C}}$. If we set $V_{ \pm}=\pi_{ \pm}^{-1}\left(V_{0}\right)$, then, by (5.1) and (5.2), we have

$$
\begin{aligned}
& V_{+}=\left\{(z, 0) \in \mathbb{H}^{n+1} \mid z \neq 0\right\} / K_{\mathbb{C}} \subset X\left(\alpha_{+}, 0\right), \\
& V_{-}=\left\{(0, w) \in \mathbb{H}^{n+1} \mid w \neq 0\right\} / K_{\mathbb{C}} \subset X\left(\alpha_{-}, 0\right),
\end{aligned}
$$

and $V_{+} \cong V_{-} \cong \mathbb{C} P^{n}$. Therefore, by (5.1) and (5.2) again, we have

$$
X\left(\alpha_{+}, 0\right) \backslash V_{+}=X\left(\alpha_{-}, 0\right) \backslash V_{-}=\left(\mu_{\mathbb{C}}^{-1}(0)^{\alpha_{+}-s s} \cap \mu_{\mathbb{C}}^{-1}(0)^{\alpha_{-}-s s}\right) / K_{\mathbb{C}}
$$

Moreover, by Lemma 3.4. we see that, if $(z, w) \in \mu_{\mathbb{C}}^{-1}(0)^{\alpha_{+}-s s} \cap \mu_{\mathbb{C}}^{-1}(0)^{\alpha_{-}-s s}$, then the orbit $(z, w) K_{\mathbb{C}}$ is closed in $\mu_{\mathbb{C}}^{-1}(0)$. Therefore $\pi_{ \pm} \mid X\left(\alpha_{ \pm}, 0\right) \backslash V_{ \pm}: X\left(\alpha_{ \pm}, 0\right) \backslash$ $V_{ \pm} \rightarrow X(0,0) \backslash V_{0}$ is a biholomorphic map. Thus we have a bimeromorphic map $f: X\left(\alpha_{+}, 0\right) \rightarrow X\left(\alpha_{-}, 0\right)$ such that $\left.f\right|_{X\left(\alpha_{+}, 0\right) \backslash V_{+}}: X\left(\alpha_{+}, 0\right) \backslash V_{+} \rightarrow X\left(\alpha_{-}, 0\right) \backslash V_{-}$ is a biholomorphic map. This is a typical example of wall-crossing phenomena and Mukai flops.

General situations are described as follows. See [Ko3 for more detailed discussions.

Theorem 6.4 Suppose that the subtorus $K$ satisfies the condition (3.6). Let $k^{*} \backslash$ $\bigcup_{s \in S_{\beta}} W_{s}$ be the chamber structure for a fixed $\beta \in k_{\mathbb{C}}^{*}$. Suppose that the chamber $\mathcal{C}_{-}$is next to the chamber $\mathcal{C}_{+}$across the wall $W_{s_{0}}$. Let $H_{s_{0}}^{(1)}$ be the 1-dimensional isotropy subgroup corresponding to the wall $W_{s_{0}}$. Set $J_{s_{0}}=\left\{i \in\{1, \ldots N\} \mid\left\langle u_{i}, Y_{s_{0}}\right\rangle \neq\right.$ $0\}$, where $Y_{s_{0}}$ is a non-zero element in LieH $_{s_{0}}^{(1)}$. Fix $\alpha_{+} \in \mathcal{C}_{+}$and $\alpha_{-} \in \mathcal{C}_{-}$. Fix 
$\alpha \in \overline{\mathcal{C}_{+}} \cap W_{s_{0}}$ such that $\alpha \notin W_{s}$ for any $s \in S_{\beta} \backslash\left\{s_{0}\right\}$. Then the following holds: (1) If we set $V_{0}=\left\{[z, w] \in X(\alpha, \beta) \mid(z, w) \zeta=(z, w)\right.$ for $\left.\zeta \in H_{s_{0}}^{(1)}\right\}$, then $V_{0}$ is a toric hyperkähler manifold.

(2) $\mu_{\mathbb{C}}^{-1}(\beta)^{\alpha_{ \pm}-s s} \subset \mu_{\mathbb{C}}^{-1}(\beta)^{\alpha-s s}$ holds respectively. So we have the natural morphisms $\pi_{ \pm}:\left(X\left(\alpha_{ \pm}, \beta\right), I_{1}\right) \rightarrow\left(X(\alpha, \beta), I_{1}\right)$.

(3) If we set $V_{ \pm}=\pi_{ \pm}^{-1}\left(V_{0}\right)$, then $\left.\pi_{ \pm}\right|_{V_{ \pm}}: V_{ \pm} \rightarrow V_{0}$ is a fiber bundle whose fiber is biholomorphic to $\mathbb{C} P^{\# J_{s_{0}}-1}$. Moreover, the codimension of $V_{ \pm}$in $X\left(\alpha_{ \pm}, \beta\right)$ is $\# J_{s_{0}}-1$, where $\# J_{s_{0}}$ is the number of elements in $J_{s_{0}}$.

(4) The natural morphism $\left.\pi_{ \pm}\right|_{X\left(\alpha_{ \pm}, \beta\right) \backslash V_{ \pm}}: X\left(\alpha_{ \pm}, \beta\right) \backslash V_{ \pm} \rightarrow X(\alpha, \beta) \backslash V_{0}$ is a biholomorphic map.

Proof. (1) Note that $W_{s_{0}}$ can be identified with the dual space of the Lie algebra $k_{s_{0}}$ of the quotient torus $K_{s_{0}}=K / H_{s_{0}}^{(1)}$. So $(\alpha, \beta)$ can be considered as an element of $k_{s_{0}}^{*} \times k_{s_{0} \mathbb{C}}^{*}$. The assumption of $\alpha$ implies $(\alpha, \beta) \in\left(k_{s_{0}}^{*} \times k_{s_{0} \mathbb{C}}^{*}\right)_{r e g}$. Then $V_{0}$ is a hyperkähler quotient of $\mathbb{H}^{\{1, \ldots, N\} \backslash J_{s_{0}}}=\left\{(z, w) \in \mathbb{H}^{N} \mid z_{i}=w_{i}=0\right.$ if $\left.i \in J_{s_{0}}\right\}$ by $K_{s_{0}}$ at $(\alpha, \beta) \in\left(k_{s_{0}}^{*} \times k_{s_{0} \mathbb{C}}^{*}\right)_{\text {reg }}$.

(2) By Lemma 3.4, it is obvious.

(3) We can choose $Y_{s_{0}} \in \operatorname{LieH}_{s_{0}}^{(1)}$ so that $\left\langle\alpha_{+}, Y_{s_{0}}\right\rangle>0$. We set $J_{s_{0}}^{+}=\left\{i \mid\left\langle u_{i}, Y_{s_{0}}\right\rangle>\right.$ $0\}$ and $J_{s_{0}}^{-}=\left\{i \mid\left\langle u_{i}, Y_{s_{0}}\right\rangle<0\right\}$ respectively. By Lemma 3.4 we can show that

$$
\mu_{\mathbb{C}}^{-1}(\beta)^{\alpha_{+}-s s}=\left\{(z, w) \in \mu_{\mathbb{C}}^{-1}(\beta)^{\alpha-s s} \mid(6.2) \text { is satisfied }\right\},
$$

where

there exists $i \in J_{s_{0}}$ such that $z_{i} \neq 0$ if $i \in J_{s_{0}}^{+}$or $w_{i} \neq 0$ if $i \in J_{s_{0}}^{-}$.

It is also easy to see that, if $(z, w) \in \mu^{-1}\left(\alpha_{+}, \beta\right)$, then $[z, w] \in V_{+}$is equivalent to

$$
w_{i}=0 \text { for } i \in J_{s_{0}}^{+} \text {and } z_{i}=0 \text { for } i \in J_{s_{0}}^{-} \text {. }
$$

Thus the fiber of $\left.\pi_{+}\right|_{V_{+}}: V_{+} \rightarrow V_{0}$ is biholomorphic to $\left(\mathbb{C}^{J_{s_{0}}} \backslash\{0\}\right) / H_{s_{0} \mathbb{C}}^{(1)}$. By the assumption that $X\left(\alpha_{+}, \beta\right)$ is non-singular, we see that the fiber is biholomorphic to $\mathbb{C} P^{\# J_{s_{0}}-1}$. Obviously the codimension of $V_{+}$in $X\left(\alpha_{+}, \beta\right)$ is $\# J_{s_{0}}-1$.

(4) By Lemma 3.4 we also have

$$
\mu_{\mathbb{C}}^{-1}(\beta)^{\alpha_{-}-s s}=\left\{(z, w) \in \mu_{\mathbb{C}}^{-1}(\beta)^{\alpha-s s} \mid \underline{6.5} \text { is satisfied }\right\},
$$

where

$$
\text { there exists } i \in J_{s_{0}} \text { such that } w_{i} \neq 0 \text { if } i \in J_{s_{0}}^{+} \text {or } z_{i} \neq 0 \text { if } i \in J_{s_{0}}^{-} .
$$

So (6.3) implies

$$
X\left(\alpha_{+}, \beta\right) \backslash V_{+}=\left(\mu_{\mathbb{C}}^{-1}(\beta)^{\alpha_{+}-s s} \cap \mu_{\mathbb{C}}^{-1}(\beta)^{\alpha_{-}-s s}\right) / K_{\mathbb{C}} .
$$

Similarly we have

$$
X\left(\alpha_{-}, \beta\right) \backslash V_{-}=\left(\mu_{\mathbb{C}}^{-1}(\beta)^{\alpha_{-}-s s} \cap \mu_{\mathbb{C}}^{-1}(\beta)^{\alpha_{+}-s s}\right) / K_{\mathbb{C}} .
$$

On the other hand, by Lemma 3.4 if $(z, w) \in \mu_{\mathbb{C}}^{-1}(\beta)^{\alpha_{+}-s s} \cap \mu_{\mathbb{C}}^{-1}(\beta)^{\alpha_{-}-s s}$, then the orbit $(z, w) K_{\mathbb{C}}$ is closed in $\mu_{\mathbb{C}}^{-1}(\beta)^{\alpha-s s}$. Thus we finish the proof.

Remark 6.5 In the above theorem we assumed the condition (3.6), that is, $X\left(\alpha_{ \pm}, \beta\right)$ is non-singular. Even if we drop this assumption, almost the same results hold. The exceptions are that $V_{0}$ is an orbifold and that the fiber of $\left.\pi_{ \pm}\right|_{V_{ \pm}}: V_{ \pm} \rightarrow V_{0}$ is a weighted projective space. The above proof works in this case. 
Proposition 6.6 Under the same assumption as Theorem 6.4 we have the following.

(1) If $\# J_{s_{0}} \geq 3$, then $\left(X\left(\alpha_{-}, \beta\right), I_{1}\right)$ is related to $\left(X\left(\alpha_{+}, \beta\right), I_{1}\right)$ by a Mukai flop.

(2) If \# $J_{s_{0}}=2$, there exists a biholomorphic map $\phi:\left(X\left(\alpha_{+}, \beta\right), I_{1}\right) \rightarrow\left(X\left(\alpha_{-}, \beta\right), I_{1}\right)$ satisfying $\pi_{+}=\pi_{-} \circ \phi$.

Proof. (1) By Theorem6.4 (3) and (4), we have a bimeromorphic map

$$
f: X\left(\alpha_{+}, \beta\right) \rightarrow X\left(\alpha_{-}, \beta\right)
$$

such that $\left.f\right|_{X\left(\alpha_{+}, \beta\right) \backslash V_{+}}: X\left(\alpha_{+}, \beta\right) \backslash V_{+} \rightarrow X\left(\alpha_{-}, \beta\right) \backslash V_{-}$is a biholomorphic map. By the proof of Theorem 6.4 (3), it is a fiber wise Mukai flop along $V_{+}$, which we also call a Mukai flop.

(2) We may assume $Y_{s_{0}}=X_{1} \pm X_{2}$. In the following we assume $Y_{s_{0}}=X_{1}+X_{2}$ and $\left\langle\alpha_{+}, Y_{s_{0}}\right\rangle>0$. (In the case $Y_{s_{0}}=X_{1}-X_{2}$, a similar argument works.)

Define a map $\widetilde{\phi}: \mu_{\mathbb{C}}^{-1}(\beta)^{\alpha_{+}-s s} \rightarrow \mu_{\mathbb{C}}^{-1}(\beta)^{\alpha_{-}-s s}$ by

$$
\widetilde{\phi}((z, w))=\left(\left(w_{2},-w_{1}, z_{3}, \ldots, z_{N}\right),\left(-z_{2}, z_{1}, w_{3}, \ldots, w_{N}\right)\right) .
$$

Suppose $(z, w) \in \mu_{\mathbb{C}}^{-1}(\beta)^{\alpha_{+}-s s}$. Since $0=\left\langle\beta, Y_{s_{0}}\right\rangle=2 \pi \sqrt{-1}\left(z_{1} w_{1}+z_{2} w_{2}\right)$, we have $\mu_{\mathbb{C}}(\widetilde{\phi}(z, w))=\mu_{\mathbb{C}}(z, w)=\beta$. By (6.1) we have $\left(z_{1}, z_{2}\right) \neq(0,0)$. Moreover, it is easy to see the following;

$$
\begin{aligned}
& \text { if }(z, w) \in \mu_{\mathbb{C}}^{-1}(\beta)^{\alpha_{+}-s s} \text { and }\left(w_{1}, w_{2}\right) \neq(0,0), \\
& \text { then there exists } t \in \mathbb{C} \text { such that } \widetilde{\phi}((z, w))=(z, w) \operatorname{Exp} t Y_{s_{0}} .
\end{aligned}
$$

Moreover, if $(z, w) \in \mu_{\mathbb{C}}^{-1}(\beta)^{\alpha_{+}-s s}$ and $\left(w_{1}, w_{2}\right)=(0,0)$, we have

$$
\alpha \in \sum_{i=3}^{N} \mathbb{R}_{\geq 0}\left|z_{i}\right|^{2} \iota^{*} u_{i}+\sum_{i=3}^{N} \mathbb{R}_{\geq 0}\left|w_{i}\right|^{2}\left(-\iota^{*} u_{i}\right) .
$$

Thus we see that $\widetilde{\phi}((z, w)) \in \mu_{\mathbb{C}}^{-1}(\beta)^{\alpha-s s}$. Moreover, by (6.4), we have $\widetilde{\phi}((z, w)) \in$ $\mu_{\mathbb{C}}^{-1}(\beta)^{\alpha_{-}-s s}$. Therefore the map $\widetilde{\phi}$ is well-defined.

Note that the map $\widetilde{\phi}$ is not $K_{\mathbb{C}^{-} \text {equivariant. However, it is easy to see that }}$ $\widetilde{\phi}\left((z, w) \operatorname{Exp} t Y_{s_{0}}\right)=(z, w) \operatorname{Exp}\left(-t Y_{s_{0}}\right)$. Moreover, if $Z \in k$ satisfies $\left\langle\iota^{*} u_{1}, Z\right\rangle=1$ and $\left\langle\iota^{*} u_{2}, Z\right\rangle=-1$, then we have $\widetilde{\phi}((z, w) \operatorname{Expt} Z)=(z, w) \operatorname{Exp} t Z$. If $Z \in k$ satisfies $\left\langle\iota^{*} u_{1}, Z\right\rangle=\left\langle\iota^{*} u_{2}, Z\right\rangle=0$, then we have $\widetilde{\phi}((z, w) \operatorname{Exp} t Z)=(z, w) \operatorname{Exp} t Z$. Thus $\widetilde{\phi}$ induces a biholomorphic map $\phi:\left(X\left(\alpha_{+}, \beta\right), I_{1}\right) \rightarrow\left(X\left(\alpha_{-}, \beta\right), I_{1}\right)$.

Finally, by (6.6) again, we have $\pi_{+}=\pi_{-} \circ \phi$.

Variation of GIT quotients was studied in [DH] and [T1]. Our cases are more restrictive, because we are treating with hyperkähler quotients. So the bimeromorphic map between two quotients is a special one, that is, a Mukai flop. Similar phenomena were observed in $\mathrm{T} 2$.

In the above proof we saw that there exists a stratification $V_{0} \subset X(\alpha, \beta)$, which comes from isotropy subgroups. More generally, if $(\alpha, \beta) \in k^{*} \times k_{\mathbb{C}}^{*} \backslash\left(k^{*} \times k_{\mathbb{C}}^{*}\right)_{\text {reg }}$, then a point $(z, w) \in \mu_{\mathbb{C}}^{-1}(\beta)$ may have an isotropy subgroup $K_{(z, w)} \subset K$ of positive dimension. So the toric hyperkähler variety $X(\alpha, \beta)$ has a stratification, which is indexed by the set of isotropy subgroups. Proudfoot and Webser used this stratification to compute the intersection cohomology of singular toric hyperkähler varieties $[\mathrm{PW}$. 


\section{References}

[Ao] Y.Aoto, On the toric hyperkähler manifolds with compact complex submanifolds, preprint.

[Au] M.Audin, Torus Actions on Symplectic Manifolds, Birkhäuser, 2004.

[Ber] M. Berger, Sur les groupes d'holonomie homogène des variétés à connexion affines et des variétés riemanniennes, Bull. Soc. Math. France 83 (1955), 279-330.

[Bi] R. Bielawski, Complete hyperkähler $4 n$-manifolds with $n$ commuting tri-Hamiltonian vector fields, Math. Ann. 314 (1999), 505-528.

[BD] R.Bielawski, A.Dancer, The Geometry and Topology of Toric Hyperkähler Manifolds, Commun. Anal. Geom. 8 (2000) 726-760.

[Do] I.Dolgachev, Lectures on Invariant Theory, Cambridge Uni. Press, 2003.

[DH] I.Dolgachev, Y.Hu, Variations of geometric invariant theory quotients, Publ.Math.de l'IHES, 87 (1998) 5-51.

[Ful] W.Fulton, Introduction to Toric Varieties, Princeton Uni. Press, 1993.

[G] V.Guillemin, Moment Maps and Combinatorial Invariants of Hamiltonian $T^{N}$-spaces, Birkhäuser, 1994.

[GS] V.Guillemin, S.Sternberg, Birational equivalence in the symplectic category, Invent. Math. 97 (1989) 485-522

[HS] T.Hausel, B.Sturmfels, Toric hyperkähler varieties, Documenta Mathematica, 7 (2002) 495534.

[HKLR] N.Hitchin, A.Karlhede, U.Lindström and M.Roček, Hyperkähler metrics and supersymmetry, Commun. Math. Phys. 108 (1987) 535-589.

[KN] G.Kempf, L.Ness, On the length of vectors in representation spaces, Lecture Notes in Math. 732 (1978) 233-242.

[Ki] F.Kirwan, Cohomology of Quotients in Symplectic and Algebraic Geometry, Princeton Uni. Press, 1984.

[Ko1] H.Konno, Cohomology rings of toric hyperKähler manifolds, Internat. J. Math. 11 (2000), 1001-1026.

[Ko2] H.Konno, On the cohomology ring of the hyperKähler analogue of the polygon spaces, Contemp. Math. 309 (2002) 129-149.

[Ko3] H.Konno, Variation of toric hyperKähler manifolds, Internat. J. Math. 14 (2003) 289-311.

[Kr] P.Kronheimer, The construction of ALE spaces as hyper-Kähler quotients, J. Diff. Geom. 29 (1989) 665-683.

[MFK] D.Mumford, J.Forgaty and F.Kirwan, Geometric Invariant Theory, Springer, 1994.

[M] S. Mukai, Symplectic structure of the moduli space of sheaves on an abelian or K3 surface, Invent. Math. 77 (1984), 101-116.

[N1] H.Nakajima, Instantons on ALE spaces, quiver varieties, and Kac-Moody algebras, Duke Math. J. 76 (1994) 365-416.

[N2] H.Nakajima, Lectures on Hilbert schemes of points on surfaces, American Math. Soc., 1999.

[P] N.Proudfoot, A survey of hypertoric geometry and topology, in this volume.

[PW] N.Proudfoot, B.Webster, Intersection cohomology of hypertoric varieties, J. Alg. Geom. 16 (2007) 39-63.

[T1] M.Thaddeus, Geometric invariant theory and flips, J. Amer. Math. Soc. 9 (1996) 691-723.

[T2] M. Thaddeus, Variation of moduli of parabolic Higgs bundles, J. Reine Angew. Math. 547 (2002), 1-14.

Graduate School of Mathematical Sciences, The University of Tokyo,

3-8-1, Komaba, Meguro-ku, Tokyo, 153-8914, Japan

E-mail address: konno@ms.u-tokyo.ac.jp 